\title{
Antiplane Shear Crack Normal to and Terminating at the Interface of Two Bonded Piezo-Electro-Magneto-Elastic Materials
}

\author{
Bogdan Rogowski \\ Department of Mechanics of Materials, Technical University of Lodz, Al. Politechniki 6, 93-590 Lodz, Poland \\ Correspondence should be addressed to Bogdan Rogowski, bogdan.rogowski@p.lodz.pl
}

Received 15 December 2011; Accepted 13 February 2012

Academic Editor: V. Sglavo

Copyright (C) 2012 Bogdan Rogowski. This is an open access article distributed under the Creative Commons Attribution License, which permits unrestricted use, distribution, and reproduction in any medium, provided the original work is properly cited.

\begin{abstract}
The magnetoelectroelastic analysis of two bonded dissimilar piezo-electro-magneto-elastic ceramics with a crack perpendicular to and terminating at the interface is made. By using the Fourier integral transform (in perpendicular directions in each materials), the mixed boundary conditions and continuity conditions are transformed to a singular integral equation with generalized Cauchy kernel, the solution of which has been well studied, and classical methods are directly applicable here to obtain the closed form solution. The results are presented for a permeable crack under anti-plane shear loading and in-plane electric and magnetic loadings, as prescribed electric displacement and magnetic inductions or electric and magnetic fields. Obtained results indicate that the magnetoelectroelastic field near the crack tip in the homogeneous PEMO-elastic ceramic is dominated by a traditional inverse square-root singularity, while the coupled field near the crack tip at the interface exhibits the singularity of power law $r^{-\alpha}$, $r$ being distant from the interface crack tip and $\alpha$ depending on the material constants of a bimaterial. In particular, electric and magnetic fields have no singularity at the crack tip in a homogeneous solid, whereas they are singular around the interface crack tip. Numerical results are given graphically to show the effects of the material properties on the singularity order, field intensity factors and energy release rates. Results presented in this paper should have potential applications to the design of multilayered magnetoelectroelastic structures.
\end{abstract}

\section{Introduction}

The newly emerging materials named magnetoelectroelasticity, which exhibit piezoelectric, piezomagnetic, and electromagnetic properties, have found increasing wide engineering applications, particularly in aerospace and automotive industries. Magnetoelectroelastic solids have been widely used as transducer, sensors, and actuators in smart structures. Because of the brittleness of PEMO-elastic materials, a high possibility of material debonding and cracking or sliding of the interface exists. Consequently, this problem has been the subject of research and discussion in the literature on elasticity theory of coupled fields. Li and Kardomateas [1] investigated the mode III interface crack problem for dissimilar piezo-electromagnetoelastic bimaterial media. The extended Stroh's theory and analytic continuation principle of complex analysis have been used to obtain the solution for interfacial cracks between two dissimilar Magnetoelectroelastic half-planes by Li and Kardomateas [2]. The problem for an antiplane interface crack between two dissimilar PEMO-elastic layers was analyzed by Wang and Mai [3]. Gao et al. [4] derived the exact solution for a permeable interface crack between two dissimilar Magnetoelectroelastic solids under general applied loads. Gao et al. [5] derived also the static solution related to antiplane crack problem. The antiplane shear cracks are a class of simple problems. But, for the case of a crack perpendicular to the interface, the problem becomes more complicated. This problem has been subject of research in the classical literature of elasticity theory. Cook and Erdogan [6] and Erdogan and Cook [7] were apparently the first to publish the solution of this problem for two bonded dissimilar isotropic half-planes. For piezoelectric biceramics an arbitrarily oriented plane crack terminating at the interface was extended by Qin and Yu [8]. The antiplane 
shear crack normal to and terminating at the interface of two piezoelectric ceramics was extended later by Li and Wang [9]. Although the above studies deal strictly with piezoelectric, it is reasonable to assume that the extension of the findings to electromagnetoelastic materials is valid.

To the best of author knowledge, the behaviour of interfacial cracks normal to and terminating at the interface of two bonded piezo-electromagnetoelastic materials has not been addressed yet. Motivated by these considerations, the author investigates the antiplane deformations and in-plane electric and magnetic fields of a PEMO-elastic bi-material with Mode-III interface crack normal to and terminating at the interface.

The crack is assumed to be electrically and magnetically permeable. Under applied electric, magnetic, and mechanical loading, electric, magnetic, and elastic behaviours near both crack tips are obtained. Two kinds of loading conditions are adopted. By using Fourier integral transform, in perpendicular directions in each materials, the associated boundary value problem is transformed to a singular integral equation with generalized Cauchy kernel. Similar types of equations have been studied, and classical methods of their solutions are directly applicable here to obtain the solution in closed form. The results indicate that magnetoelectroelastic field near the crack tip in a homogeneous PEMO-elastic ceramic exhibits an inverse square-root singularity, while singular field near the interface crack tip is dominant by a singularity of power law. The singularity order is dependent on relevant $2 \times 6$ material constants of two ceramics. The effects of magneto-electro-mechanical parameters on the field intensity factors are evaluated by numerical analysis, which could be of particular interest to the analysis and design of smart sensors/actuators constructed from Magnetoelectroelastic composite laminates.

\section{Formulation of the Problem}

2.1. Basic Equations. For a linearly Magnetoelectroelastic medium under antiplane shear coupled with in-plane electric and magnetic fields, there are only the nontrivial antiplane displacement $w$ :

$$
u_{x}=0, \quad u_{y}=0, \quad u_{z}=w(x, y),
$$

strain components $\gamma_{x z}$ and $\gamma_{y z}$ :

$$
\gamma_{x z}=\frac{\partial w}{\partial x}, \quad \gamma_{y z}=\frac{\partial w}{\partial y}
$$

stress components $\tau_{x z}$ and $\tau_{y z}$, in-plane electrical and magnetic potentials $\phi$ and $\psi$, which define electric and magnetic field components $E_{x}, E_{y}, H_{x}$, and $H_{y}$ :

$$
E_{x}=-\frac{\partial \phi}{\partial x}, \quad E_{y}=-\frac{\partial \phi}{\partial y}, \quad H_{x}=-\frac{\partial \psi}{\partial x}, \quad H_{y}=-\frac{\partial \psi}{\partial y}
$$

and electrical displacement components $D_{x}, D_{y}$, and magnetic induction components $B_{x}$, and $B_{y}$ with all field quantities being the functions of coordinates $x$ and $y$.
The relations (2) and (3) have the following form:

$$
\gamma_{\alpha z}=w_{, \alpha}, \quad E_{\alpha}=-\phi_{, \alpha}, \quad H_{\alpha}=-\psi_{, \alpha},
$$

where $\alpha=x, y$ and $w_{, \alpha}=\partial w / \partial \alpha$.

For linearly Magnetoelectroelastic medium, the coupled constitutive relations can be written in the matrix form as follows:

$$
\left[\tau_{\alpha z}, D_{\alpha}, B_{\alpha}\right]^{T}=\mathrm{C}\left[\gamma_{\alpha z},-E_{\alpha},-H_{\alpha}\right]^{T} \text {, }
$$

where the superscript $T$ denotes the transpose of a matrix and

$$
\mathrm{C}=\left[\begin{array}{ccc}
c_{44} & e_{15} & q_{15} \\
e_{15} & -\varepsilon_{11} & -d_{11} \\
q_{15} & -d_{11} & -\mu_{11}
\end{array}\right],
$$

where $c_{44}$ is the shear modulus along the $z$-direction, which is direction of poling and is perpendicular to the isotropic plane $(x, y), \varepsilon_{11}$ and $\mu_{11}$ are dielectric permittivity, and magnetic permeability coefficients, respectively, $e_{15}, q_{15}$, and $d_{11}$ are piezoelectric, piezomagnetic and magneto-electric coefficients, respectively.

The mechanical equilibrium equation (called as Euler equation) and the charge and current conservation equations (called as Maxwell equations), in the absence of the body force electric and magnetic charge densities, can be written as

$$
\tau_{z \alpha, \alpha}=0, \quad D_{\alpha, \alpha}=0, \quad B_{\alpha, \alpha}=0, \quad \alpha=x, y .
$$

Subsequently, the Euler and Maxwell equations take the following form:

$$
\mathrm{C}\left[\nabla^{2} w, \nabla^{2} \phi, \nabla^{2} \psi\right]^{T}=[0,0,0]^{T},
$$

where $\nabla^{2}=\partial^{2} / \partial x^{2}+\partial^{2} / \partial y^{2}$ is the two-dimensional Laplace operator.

Since $|C| \neq 0$, one can decouple the $(8)$ as follows:

$$
\nabla^{2} w=0, \quad \nabla^{2} \phi=0, \quad \nabla^{2} \psi=0 .
$$

If we introduce, for convenience of mathematics in some boundary value problems, two unknown functions

$$
\left[\chi-e_{15} w, \eta-q_{15} w\right]^{T}=\mathrm{C}_{0}[\phi, \psi]^{T},
$$

where

$$
\mathrm{C}_{0}=\left[\begin{array}{cc}
-\varepsilon_{11} & -d_{11} \\
-d_{11} & -\mu_{11}
\end{array}\right]
$$

then

$$
[\phi, \psi]^{T}=\mathrm{C}_{0}^{-1}\left[\chi-e_{15} w, \eta-q_{15} w\right]^{T}
$$

where

$$
\mathrm{C}_{0}^{-1}=\frac{1}{\varepsilon_{11} \mu_{11}-d_{11}^{2}}\left[\begin{array}{cc}
-\mu_{11} & d_{11} \\
d_{11} & -\varepsilon_{11}
\end{array}\right]=\left[\begin{array}{ll}
e_{1} & e_{2} \\
e_{2} & e_{3}
\end{array}\right] .
$$


The governing field variables are

$$
\begin{gathered}
\tau_{z k}=\tilde{c}_{44} w_{, k}-\alpha D_{k}-\beta B_{k}, \\
\phi=\alpha w+e_{1} \chi+e_{2} \eta, \\
\psi=\beta w+e_{2} \chi+e_{3} \eta, \\
D_{k}=\chi, k, \\
B_{k}=\eta_{, k}, \quad k=x, y, \\
\nabla^{2} w=0, \quad \nabla^{2} \chi=0, \quad \nabla^{2} \eta=0,
\end{gathered}
$$

where

$$
\begin{gathered}
\tilde{c}_{44}=c_{44}+\alpha e_{15}+\beta q_{15}, \\
\alpha=\frac{\mu_{11} e_{15}-d_{11} q_{15}}{\varepsilon_{11} \mu_{11}-d_{11}^{2}}=-\left(e_{1} e_{15}+e_{2} q_{15}\right), \\
\beta=\frac{\varepsilon_{11} q_{15}-d_{11} e_{15}}{\varepsilon_{11} \mu_{11}-d_{11}^{2}}=-\left(e_{3} q_{15}+e_{2} e_{15}\right) .
\end{gathered}
$$

Note that $\tilde{c}_{44}$ is the piezo-electro-magnetically stiffened elastic constant.

Note also that the inverse of a matrix $\mathrm{C}$ is defined by parameters $\alpha, \beta, \tilde{c}_{44}$ and $e_{1}, e_{2}, e_{3}$ as follows:

$$
\mathrm{C}^{-1}=\frac{1}{\widetilde{c}_{44}}\left[\begin{array}{ccc}
1 & \alpha & \beta \\
\alpha & \alpha^{2}+\tilde{c}_{44} e_{1} & \alpha \beta+\tilde{c}_{44} e_{2} \\
\beta & \alpha \beta+\tilde{c}_{44} e_{2} & \beta^{2}+\tilde{c}_{44} e_{3}
\end{array}\right] .
$$

These material parameters will appear in our solutions.

2.2. Boundary Conditions. Consider a crack terminating at the interface of two bonded dissimilar PEMO-elastic ceramics polarized in the $z$ direction. For convenience, we denote the PEMO-elastic ceramics occupying the right and left half-planes $x \geq 0$ and $x \leq 0$ as piezoceramics I and II, respectively, shown in Figure 1.

Let a crack be perpendicular to the interface and be situated at $[0, a](a>0)$ in the positive $x$-direction in ceramic I. For an antiplane shear crack having no thickness (so-called "mathematical crack"), the crack surfaces contact each other, in reality; so the crack is electrically and magnetically contacted. Consequently, the electric and magnetic boundary conditions at the crack surfaces can be described according to so-called double permeable conditions, namely,

$$
\begin{array}{cc}
D_{y}\left(x, 0^{+}\right)=D_{y}\left(x, 0^{-}\right), & B_{y}\left(x, 0^{+}\right)=B_{y}\left(x, 0^{-}\right), \\
\phi\left(x, 0^{+}\right)=\phi\left(x, 0^{-}\right), & \psi\left(x, 0^{+}\right)=\psi\left(x, 0^{-}\right) .
\end{array}
$$

Note that besides the crack surfaces, the above conditions, in fact, certainly hold at the crack-absent parts of the crack plane. Using the relations (14), it can be shown that the condition (18) may be replaced by conditions as follows:

$$
\begin{gathered}
\chi, y\left(x, 0^{+}\right)=\chi_{, y}\left(x, 0^{-}\right), \quad \eta_{, y}\left(x, 0^{+}\right)=\eta_{, y}\left(x, 0^{-}\right), \\
\chi=e_{15} w, \quad \eta=q_{15} w \quad \text { for } x, y=0 \pm .
\end{gathered}
$$

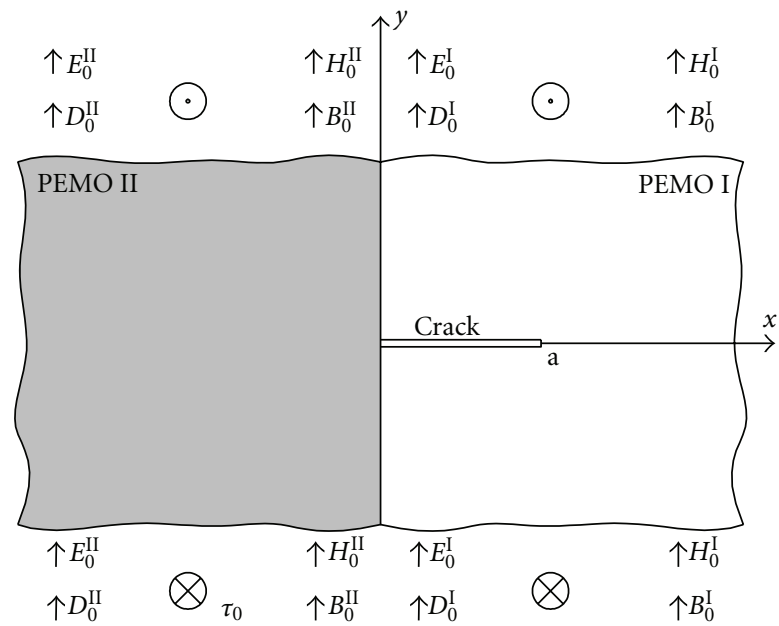

Figure 1: Two bonded dissimilar PEMO-elastic ceramics with a crack perpendicular to and terminating at the interface.

Let the constant mechanical loads and uniform electric displacement and magnetic induction or electric field and magnetic field be applied at infinity (two cases of electric and magnetic loads), and the following:

$$
\begin{aligned}
& \tau_{y z}^{\mathrm{I}}(x, y)=\tau_{0}^{\mathrm{I}}, \quad D_{y}^{\mathrm{I}}(x, y)=D_{0}^{\mathrm{I}}, \quad B_{y}^{\mathrm{I}}(x, y)=B_{0}^{\mathrm{I}} \\
& \text { or } E_{y}^{\mathrm{I}}(x, y)=E_{0}^{\mathrm{I}}, \quad H_{y}^{\mathrm{I}}(x, y)=H_{0}^{\mathrm{I}}, \quad x>0, \quad y \longrightarrow \pm \infty \\
& \tau_{y z}^{\mathrm{II}}(x, y)=\tau_{0}^{\mathrm{II}}, \quad D_{y}^{\mathrm{II}}(x, y)=D_{0}^{\mathrm{II}}, \quad B_{y}^{\mathrm{II}}(x, y)=B_{0}^{\mathrm{II}} \\
& \text { or } E_{y}^{\mathrm{II}}(x, y)=E_{0}^{\mathrm{II}}, \quad H_{y}^{\mathrm{II}}(x, y)=H_{0}^{\mathrm{II}}, \quad x<0, \quad y \longrightarrow \pm \infty,
\end{aligned}
$$

where $\tau_{0}^{\mathrm{I}}\left(\tau_{0}^{\mathrm{II}}\right), D_{0}^{\mathrm{I}}\left(D_{0}^{\mathrm{II}}\right), B_{0}^{\mathrm{I}}\left(B_{0}^{\mathrm{II}}\right)$ or $E_{0}^{\mathrm{I}}\left(E_{0}^{\mathrm{II}}\right), H_{0}^{\mathrm{I}}\left(H_{0}^{\mathrm{II}}\right)$ are prescribed constants, a quantity with superscribes I or II that specifies the one in the PEMO-ceramic I or II, respectively. To solve the crack problem in linear elastic solids, the superposition technique is usually used. Thus, we first solve the Magnetoelectroelastic field problem without the cracks in the medium under electric, magnetic, and mechanical loads. This elementary solution is the following:

$$
\begin{aligned}
& \tau_{y z}^{J}=\tau_{0}^{J}, \\
& D_{y}^{J}=D^{J}=\left\{\begin{array}{rr}
D_{0}^{J}, & \text { case I } \\
{\left[\frac{e_{15}}{c_{44}} \tau_{0}+\left(\varepsilon_{11}+\frac{e_{15}^{2}}{c_{44}}\right) E_{0}\right.} & \\
\left.+\left(d_{11}+\frac{e_{15} q_{15}}{c_{44}}\right) H_{0}\right]^{J}, & \text { case II }
\end{array}\right.
\end{aligned}
$$

$$
B_{y}^{J}=B^{J}=\left\{\begin{array}{l}
B_{0}^{J}, \\
{\left[\frac{q_{15}}{c_{44}} \tau_{0}+\left(d_{11}+\frac{e_{15} q_{15}}{c_{44}}\right) E_{0}\right.} \\
\left.+\left(\mu_{11}+\frac{q_{15}^{2}}{c_{44}}\right) H_{0}\right]^{J},
\end{array}\right.
$$

case I

with $J=$ I, II. 
In addition the crack surfaces are traction-free, that is,

$$
\tau_{y z}^{\mathrm{I}}(x, y)=0 ; \quad y=0 \pm, 0<x<a,
$$

and owing to the symmetry one can directly write following conditions:

$$
w^{\mathrm{I}}(x, 0)=0, \quad x>a, \quad w^{\mathrm{II}}(x, 0)=0, \quad x<0 .
$$

We further consider the situation when the interface under consideration is perfectly bonded, across which the displacement, stress, electric and magnetic potentials, electric displacement, and magnetic induction are continuous

$$
\begin{array}{lll}
w^{\mathrm{I}}(0, y)=w^{\mathrm{II}}(0, y), & \tau_{x z}^{\mathrm{I}}(0, y)=\tau_{x z}^{\mathrm{II}}(0, y) ; & -\infty<y<\infty, \\
\phi^{\mathrm{I}}(0, y)=\phi^{\mathrm{II}}(0, y), & D_{x}^{\mathrm{I}}(0, y)=D_{x}^{\mathrm{II}}(0, y) ; & -\infty<y<\infty, \\
\psi^{\mathrm{I}}(0, y)=\psi^{\mathrm{II}}(0, y), & B_{x}^{\mathrm{I}}(0, y)=B_{x}^{\mathrm{II}}(0, y) ; & -\infty<y<\infty .
\end{array}
$$

\section{Method of Solution}

From the symmetry of the problem, it is sufficient to consider the upper half-plane of the bi-ceramic. Consequently, for $y \geq 0$, it is easily found that an appropriate solution of the problem, which satisfies the boundary conditions (19a) and (20), takes of the following form:

$$
\begin{aligned}
{\left[\begin{array}{l}
w^{\mathrm{I}}(x, y) \\
\chi^{\mathrm{I}}(x, y) \\
\eta^{\mathrm{I}}(x, y)
\end{array}\right]=} & {\left[\begin{array}{l}
\gamma^{\mathrm{I}} \\
D^{\mathrm{I}} \\
B^{\mathrm{I}}
\end{array}\right] y+\left[\begin{array}{c}
1 \\
e_{15}^{\mathrm{I}} \\
q_{15}^{\mathrm{I}}
\end{array}\right] \int_{0}^{\infty} A_{1}(\xi) e^{-y \xi} \cos (\xi x) d \xi } \\
& +\int_{0}^{\infty}\left[\begin{array}{l}
B_{1}(\xi) \\
C_{1}(\xi) \\
D_{1}(\xi)
\end{array}\right] e^{-\xi x} \sin (\xi y) d \xi,
\end{aligned}
$$

for $x \geq 0$ and

$$
\begin{aligned}
{\left[\begin{array}{c}
w^{\mathrm{II}}(x, y) \\
\chi^{\mathrm{II}}(x, y) \\
\eta^{\mathrm{II}}(x, y)
\end{array}\right]=} & {\left[\begin{array}{c}
\gamma^{\mathrm{II}} \\
D^{\mathrm{II}} \\
B^{\mathrm{II}}
\end{array}\right] y+\left[\begin{array}{c}
1 \\
e_{15}^{\mathrm{II}} \\
q_{15}^{\mathrm{II}}
\end{array}\right] \int_{0}^{\infty} A_{2}(\xi) e^{-y \xi} \cos (\xi x) d \xi } \\
& +\int_{0}^{\infty}\left[\begin{array}{l}
B_{2}(\xi) \\
C_{2}(\xi) \\
D_{2}(\xi)
\end{array}\right] e^{\xi x} \sin (\xi y) d \xi
\end{aligned}
$$

for $x \leq 0$, where $A_{j}, B_{j}, C_{j}$, and $D_{j}(j=1,2)$ are unknowns to be determined from given boundary conditions and where

$$
\gamma^{J}=\frac{\tau_{0}^{J}+\alpha^{J} D^{J}+\beta^{J} B^{J}}{\tilde{c}_{44}^{J}} ; \quad J=\text { I, II. }
$$

Furthermore with the aid of (14), one can give the components of stress, electric displacement, magnetic induction, and electric and magnetic potentials

$$
\begin{aligned}
{\left[\begin{array}{c}
\tau_{y z}^{\mathrm{I}}(x, y) \\
D_{y}^{\mathrm{I}}(x, y) \\
B_{y}^{\mathrm{I}}(x, y)
\end{array}\right]=} & {\left[\begin{array}{c}
\tau_{0}^{\mathrm{I}} \\
D^{\mathrm{I}} \\
B^{\mathrm{I}}
\end{array}\right]-\left[\begin{array}{c}
c_{44}^{\mathrm{I}} \\
e_{15}^{\mathrm{I}} \\
q_{15}^{\mathrm{I}}
\end{array}\right] \int_{0}^{\infty} \xi A_{1}(\xi) e^{-y \xi} \cos (\xi x) d \xi } \\
& +\int_{0}^{\infty} \xi\left[\begin{array}{c}
\tilde{c}_{44}^{\mathrm{I}} B_{1}(\xi)-\alpha^{\mathrm{I}} C_{1}(\xi)-\beta^{\mathrm{I}} D_{1}(\xi) \\
C_{1}(\xi) \\
D_{1}(\xi)
\end{array}\right] \\
& \times e^{-\xi x} \cos (\xi y) d \xi
\end{aligned}
$$

$$
\begin{aligned}
{\left[\begin{array}{c}
\phi^{\mathrm{I}}(x, y) \\
\psi^{\mathrm{I}}(x, y)
\end{array}\right]=C_{\mathrm{I}}^{-1} } & {\left[\begin{array}{c}
\tau_{0}^{\mathrm{I}} \\
D^{\mathrm{I}} \\
B^{\mathrm{I}}
\end{array}\right] y+0+\left[\begin{array}{lll}
\alpha & e_{1} & e_{2} \\
\beta & e_{2} & e_{3}
\end{array}\right]^{\mathrm{I}} } \\
& \times \int_{0}^{\infty} \xi\left[\begin{array}{l}
B_{1}(\xi) \\
C_{1}(\xi) \\
D_{1}(\xi)
\end{array}\right] e^{-\xi x} \sin (\xi y) d \xi
\end{aligned}
$$

for $x \geq 0$ and

$$
\begin{aligned}
{\left[\begin{array}{c}
\tau_{y z}^{\mathrm{II}}(x, y) \\
D_{y}^{\mathrm{II}}(x, y) \\
B_{y}^{\mathrm{II}}(x, y)
\end{array}\right]=} & {\left[\begin{array}{c}
\tau_{0}^{\mathrm{II}} \\
D^{\mathrm{II}} \\
B^{\mathrm{II}}
\end{array}\right]-\left[\begin{array}{c}
c_{44}^{\mathrm{II}} \\
e_{15}^{\mathrm{II}} \\
q_{15}^{\mathrm{II}}
\end{array}\right] \int_{0}^{\infty} \xi A_{2}(\xi) e^{-y \xi} \cos (\xi x) d \xi } \\
& +\int_{0}^{\infty} \xi\left[\begin{array}{c}
\tilde{c}_{44}^{\mathrm{II}} B_{1}(\xi)-\alpha^{\mathrm{II}} C_{2}(\xi)-\beta^{\mathrm{II}} D_{2}(\xi) \\
C_{2}(\xi) \\
D_{2}(\xi)
\end{array}\right] \\
& \times e^{\xi x} \cos (\xi y) d \xi,
\end{aligned}
$$$$
\left[\begin{array}{l}
\phi^{\mathrm{II}}(x, y) \\
\psi^{\mathrm{II}}(x, y)
\end{array}\right]=C_{\mathrm{II}}^{-1}\left[\begin{array}{c}
\tau_{0}^{\mathrm{II}} \\
D^{\mathrm{II}} \\
B^{\mathrm{II}}
\end{array}\right] y+0+\left[\begin{array}{lll}
\alpha & e_{1} & e_{2} \\
\beta & e_{2} & e_{3}
\end{array}\right]^{\mathrm{II}}
$$$$
\times \int_{0}^{\infty} \xi\left[\begin{array}{l}
B_{2}(\xi) \\
C_{2}(\xi) \\
D_{2}(\xi)
\end{array}\right] e^{\xi x} \sin (\xi y) d \xi
$$ 
for $x \leq 0$ and

$$
\begin{aligned}
{\left[\begin{array}{c}
\tau_{x z}^{\mathrm{I}}(x, y) \\
D_{x}^{\mathrm{I}}(x, y) \\
B_{x}^{\mathrm{I}}(x, y)
\end{array}\right]=} & -\left[\begin{array}{c}
c_{44}^{\mathrm{I}} \\
e_{15}^{\mathrm{I}} \\
q_{15}^{\mathrm{I}}
\end{array}\right] \int_{0}^{\infty} \xi A_{1}(\xi) e^{-y \xi} \sin (\xi x) d \xi \\
& -\int_{0}^{\infty} \xi\left[\begin{array}{c}
\tilde{c}_{44}^{\mathrm{I}} B_{1}(\xi)-\alpha^{\mathrm{I}} C_{1}(\xi)-\beta^{\mathrm{I}} D_{1}(\xi) \\
C_{1}(\xi) \\
D_{1}(\xi)
\end{array}\right] \\
& \times e^{-\xi x} \sin (\xi y) d \xi
\end{aligned}
$$

for $x \geq 0$ and

$$
\begin{aligned}
{\left[\begin{array}{c}
\tau_{x z}^{\mathrm{II}}(x, y) \\
D_{x}^{\mathrm{II}}(x, y) \\
B_{x}^{\mathrm{II}}(x, y)
\end{array}\right]=} & -\left[\begin{array}{c}
c_{44}^{\mathrm{II}} \\
e_{15}^{\mathrm{II}} \\
q_{15}^{\mathrm{II}}
\end{array}\right] \int_{0}^{\infty} \xi A_{2}(\xi) e^{-y \xi} \sin (\xi x) d \xi \\
& +\int_{0}^{\infty} \xi\left[\begin{array}{c}
\widetilde{c}_{44}^{\mathrm{II}} B_{2}(\xi)-\alpha^{\mathrm{II}} C_{2}(\xi)-\beta^{\mathrm{II}} D_{2}(\xi) \\
C_{2}(\xi) \\
D_{2}(\xi)
\end{array}\right] \\
& \times e^{\xi x} \sin (\xi y) d \xi
\end{aligned}
$$

for $x \leq 0$.

Now, application of the continuity conditions (24), at the interface $x=0$ to (25) to (33), yields

$$
\begin{gathered}
\frac{\tau_{0}^{\mathrm{I}}+\alpha^{\mathrm{I}} D^{\mathrm{I}}+\beta^{\mathrm{I}} B^{\mathrm{I}}}{\tilde{c}_{44}^{\mathrm{I}}}=\frac{\tau_{0}^{\mathrm{II}}+\alpha^{\mathrm{II}} D^{\mathrm{II}}+\beta^{\mathrm{II}} B^{\mathrm{II}}}{\tilde{c}_{44}^{\mathrm{I}}}, \\
C_{\mathrm{I}}^{-1}\left[\begin{array}{c}
\tau_{0}^{\mathrm{I}} \\
D^{\mathrm{I}} \\
B^{\mathrm{I}}
\end{array}\right]=C_{\mathrm{II}}^{-1}\left[\begin{array}{c}
\tau_{0}^{\mathrm{II}} \\
D^{\mathrm{II}} \\
B^{\mathrm{II}}
\end{array}\right], \\
C_{J}^{-1}=\frac{1}{\widetilde{c}_{44}^{J}}\left[\begin{array}{lll}
\alpha & \alpha^{2}+\tilde{c}_{44}^{\mathrm{I}} e_{1} & \alpha \beta+\tilde{c}_{44}^{\mathrm{I}} e_{2} \\
\beta & \alpha \beta+\tilde{c}_{44}^{\mathrm{I}} e_{2} & \beta^{2}+\tilde{c}_{44}^{\mathrm{I}} e_{3}
\end{array}\right]
\end{gathered}
$$

$$
\int_{0}^{\infty} e^{-\eta y} \sin (\xi y) d y=\frac{\xi}{\xi^{2}+\eta^{2}} .
$$

The result (40) in connection with (36) and (37) yields

$$
\begin{aligned}
B_{1}(\xi)= & -\frac{2}{\pi} \mathrm{I} \frac{\tilde{c}_{44}^{\mathrm{II}} \Delta+\left(e_{3}^{\mathrm{II}}+e_{3}^{\mathrm{I}}\right) \alpha^{\mathrm{II}}\left(\alpha^{\mathrm{II}}-\alpha^{\mathrm{I}}\right)+\left(e_{1}^{\mathrm{II}}+e_{1}^{\mathrm{I}}\right) \beta^{\mathrm{II}}\left(\beta^{\mathrm{II}}-\beta^{\mathrm{I}}\right)}{\left(\tilde{c}_{44}^{\mathrm{I}}+\tilde{c}_{44}^{\mathrm{II}}\right) \Delta+\left(e_{3}^{\mathrm{II}}+e_{3}^{\mathrm{I}}\right)\left(\alpha^{\mathrm{II}}-\alpha^{\mathrm{I}}\right)^{2}+\left(e_{1}^{\mathrm{II}}+e_{1}^{\mathrm{I}}\right)\left(\beta^{\mathrm{II}}-\beta^{\mathrm{I}}\right)^{2}} \\
& +\frac{2}{\pi} \mathrm{I} \frac{\left(e_{2}^{\mathrm{II}}+e_{2}^{\mathrm{I}}\right)\left(\alpha^{\mathrm{I}} \beta^{\mathrm{II}}+\beta^{\mathrm{I}} \alpha^{\mathrm{II}}+2 \alpha^{\mathrm{II}} \beta^{\mathrm{II}}\right)}{\left(\tilde{c}_{44}^{\mathrm{I}}+\tilde{c}_{44}^{\mathrm{II}}\right) \Delta+\left(e_{3}^{\mathrm{II}}+e_{3}^{\mathrm{I}}\right)\left(\alpha^{\mathrm{II}}-\alpha^{\mathrm{I}}\right)^{2}+\left(e_{1}^{\mathrm{II}}+e_{1}^{\mathrm{I}}\right)\left(\beta^{\mathrm{II}}-\beta^{\mathrm{I}}\right)^{2}}, \\
B_{2}(\xi)= & \frac{2}{\pi} \mathrm{I} \frac{\tilde{c}_{44}^{\mathrm{I}} \Delta-\left(e_{3}^{\mathrm{II}}+e_{3}^{\mathrm{I}}\right) \alpha^{\mathrm{I}}\left(\alpha^{\mathrm{II}}-\alpha^{\mathrm{I}}\right)-\left(e_{1}^{\mathrm{II}}+e_{1}^{\mathrm{I}}\right) \beta^{\mathrm{I}}\left(\beta^{\mathrm{II}}-\beta^{\mathrm{I}}\right)}{\left(\tilde{c}_{44}^{\mathrm{I}}+\tilde{c}_{44}^{\mathrm{II}}\right) \Delta+\left(e_{3}^{\mathrm{II}}+e_{3}^{\mathrm{I}}\right)\left(\alpha^{\mathrm{II}}-\alpha^{\mathrm{I}}\right)^{2}+\left(e_{1}^{\mathrm{II}}+e_{1}^{\mathrm{I}}\right)\left(\beta^{\mathrm{II}}-\beta^{\mathrm{I}}\right)^{2}} \\
& +\frac{2}{\pi} \mathrm{I} \frac{\left(e_{2}^{\mathrm{II}}+e_{2}^{\mathrm{I}}\right)\left(\alpha^{\mathrm{I}} \beta^{\mathrm{II}}+\beta^{\mathrm{I}} \alpha^{\mathrm{II}}+2 \alpha^{\mathrm{II}} \beta^{\mathrm{II}}\right)}{\left(\tilde{c}_{44}^{\mathrm{I}}+\tilde{c}_{44}^{\mathrm{II}}\right) \Delta+\left(e_{3}^{\mathrm{II}}+e_{3}^{\mathrm{I}}\right)\left(\alpha^{\mathrm{II}}-\alpha^{\mathrm{I}}\right)^{2}+\left(e_{1}^{\mathrm{II}}+e_{1}^{\mathrm{I}}\right)\left(\beta^{\mathrm{II}}-\beta^{\mathrm{I}}\right)^{2}},
\end{aligned}
$$




$$
\begin{aligned}
& C_{1}(\xi)=B_{1}(\xi) \frac{\beta^{\mathrm{I}}\left(e_{2}^{\mathrm{II}}+e_{2}^{\mathrm{I}}\right)-\alpha^{\mathrm{I}}\left(e_{3}^{\mathrm{II}}+e_{3}^{\mathrm{I}}\right)}{\Delta}-B_{2}(\xi) \frac{\beta^{\mathrm{II}}\left(e_{2}^{\mathrm{II}}+e_{2}^{\mathrm{I}}\right)-\alpha^{\mathrm{II}}\left(e_{3}^{\mathrm{II}}+e_{3}^{\mathrm{I}}\right)}{\Delta}, \\
& D_{1}(\xi)=-B_{1}(\xi) \frac{\beta^{\mathrm{I}}\left(e_{1}^{\mathrm{II}}+e_{1}^{\mathrm{I}}\right)-\alpha^{\mathrm{I}}\left(e_{2}^{\mathrm{II}}+e_{2}^{\mathrm{I}}\right)}{\Delta}+B_{2}(\xi) \frac{\beta^{\mathrm{II}}\left(e_{1}^{\mathrm{II}}+e_{1}^{\mathrm{I}}\right)-\alpha^{\mathrm{II}}\left(e_{2}^{\mathrm{II}}+e_{2}^{\mathrm{I}}\right)}{\Delta},
\end{aligned}
$$

where

$$
\begin{gathered}
\Delta=\left(e_{1}^{\mathrm{II}}+e_{1}^{\mathrm{I}}\right)\left(e_{3}^{\mathrm{II}}+e_{3}^{\mathrm{I}}\right)-\left(e_{2}^{\mathrm{II}}+e_{2}^{\mathrm{I}}\right)^{2}, \\
\mathrm{I}=\int_{0}^{\infty} A_{1}(\eta) \frac{\xi}{\xi^{2}+\eta^{2}} d \eta
\end{gathered}
$$

In the special cases, we obtain that for both piezoelectric materials

$$
\begin{gathered}
B_{1}(\xi)=-\frac{2}{\pi} \mathrm{I} \frac{c_{44}^{\mathrm{II}}\left(\varepsilon_{11}^{\mathrm{I}}+\varepsilon_{11}^{\mathrm{II}}\right)+e_{15}^{\mathrm{II}}\left(e_{15}^{\mathrm{I}}+e_{15}^{\mathrm{II}}\right)}{\left(c_{44}^{\mathrm{I}}+c_{44}^{\mathrm{II}}\right)\left(\varepsilon_{11}^{\mathrm{I}}+\varepsilon_{11}^{\mathrm{II}}\right)+\left(e_{15}^{\mathrm{I}}+e_{15}^{\mathrm{II}}\right)^{2}}, \\
B_{2}(\xi)=\frac{2}{\pi} \mathrm{I} \frac{c_{44}^{\mathrm{I}}\left(\varepsilon_{11}^{\mathrm{I}}+\varepsilon_{11}^{\mathrm{II}}\right)+e_{15}^{\mathrm{I}}\left(e_{15}^{\mathrm{I}}+e_{15}^{\mathrm{II}}\right)}{\left(c_{44}^{\mathrm{I}}+c_{44}^{\mathrm{II}}\right)\left(\varepsilon_{11}^{\mathrm{I}}+\varepsilon_{11}^{\mathrm{II}}\right)+\left(e_{15}^{\mathrm{I}}+e_{15}^{\mathrm{II}}\right)^{2}}, \\
C_{1}(\xi)=-\frac{2}{\pi} \mathrm{I} \frac{e_{15}^{\mathrm{I}}\left(c_{44}^{\mathrm{II}} \varepsilon_{11}^{\mathrm{II}}+\left(e_{15}^{\mathrm{II}}\right)^{2}\right)+e_{15}^{\mathrm{II}}\left(c_{44}^{\mathrm{I}} \varepsilon_{11}^{\mathrm{I}}+\left(e_{15}^{\mathrm{I}}\right)^{2}\right)}{\left(c_{44}^{\mathrm{I}}+c_{44}^{\mathrm{II}}\right)\left(\varepsilon_{11}^{\mathrm{I}}+\varepsilon_{11}^{\mathrm{II}}\right)+\left(e_{15}^{\mathrm{I}}+e_{15}^{\mathrm{II}}\right)^{2}}, \\
D_{1}(\xi)=0,
\end{gathered}
$$

for both piezomagnetic materials

$$
\begin{gathered}
B_{1}(\xi)=-\frac{2}{\pi} \mathrm{I} \frac{c_{44}^{\mathrm{II}}\left(\mu_{11}^{\mathrm{I}}+\mu_{11}^{\mathrm{II}}\right)+q_{15}^{\mathrm{II}}\left(q_{15}^{\mathrm{I}}+q_{15}^{\mathrm{II}}\right)}{\left(c_{44}^{\mathrm{I}}+c_{44}^{\mathrm{II}}\right)\left(\mu_{11}^{\mathrm{I}}+\mu_{11}^{\mathrm{II}}\right)+\left(q_{15}^{\mathrm{I}}+q_{15}^{\mathrm{II}}\right)^{2}}, \\
B_{2}(\xi)=\frac{2}{\pi} \mathrm{I} \frac{c_{44}^{\mathrm{I}}\left(\mu_{11}^{\mathrm{I}}+\mu_{11}^{\mathrm{II}}\right)+q_{15}^{\mathrm{I}}\left(q_{15}^{\mathrm{I}}+q_{15}^{\mathrm{II}}\right)}{\left(c_{44}^{\mathrm{I}}+c_{44}^{\mathrm{II}}\right)\left(\mu_{11}^{\mathrm{I}}+\mu_{11}^{\mathrm{II}}\right)+\left(q_{15}^{\mathrm{I}}+q_{15}^{\mathrm{II}}\right)^{2}}, \\
C_{1}(\xi)=0, \\
D_{1}(\xi)=-\frac{2}{\pi} \mathrm{I} \frac{q_{15}^{\mathrm{I}}\left(c_{44}^{\mathrm{II}} \mu_{11}^{\mathrm{II}}+\left(q_{15}^{\mathrm{II}}\right)^{2}\right)+q_{15}^{\mathrm{II}}\left(c_{44}^{\mathrm{I}} \mu_{11}^{\mathrm{I}}+\left(q_{15}^{\mathrm{I}}\right)^{2}\right)}{\left(c_{44}^{\mathrm{I}}+c_{44}^{\mathrm{II}}\right)\left(\mu_{11}^{\mathrm{I}}+\mu_{11}^{\mathrm{II}}\right)+\left(q_{15}^{\mathrm{I}}+q_{15}^{\mathrm{II}}\right)^{2}} .
\end{gathered}
$$

The formulae (45) are equivalent to that derived by Li and Wang [9] who solved the problem of two bonded dissimilar piezoelectric media with an antiplane shear crack perpendicular to and terminated at the interface. Next, we denote that

$$
g(x)=\frac{\partial w^{\mathrm{I}}\left(x, 0^{+}\right)}{\partial x}
$$

From the boundary conditions (23), $g(x)$ should satisfy the single-value displacement constraint condition, that is,

$$
\int_{0}^{a} g(x) d x=0
$$

Utilizing $(25)_{1}$ in (23) leads to

$$
w^{\mathrm{I}}(x, 0)=\int_{0}^{\infty} A_{1}(\xi) \cos (\xi x) d \xi=0, \quad x>a
$$

from which together with (47), by using the inverse Fourier transform, can be deduced

$$
A_{1}(\xi)=-\frac{2}{\pi \xi} \int_{0}^{a} g(t) \sin (\xi t) d t .
$$

Now, we calculate the following:

$$
\int_{0}^{\infty} \frac{\xi}{\xi^{2}+\eta^{2}} A_{1}(\eta) d \eta=-\frac{2}{\pi} \xi \int_{0}^{a} g(t) d t \int_{0}^{\infty} \frac{\sin (\eta t)}{\eta\left(\xi^{2}+\eta^{2}\right)} d \eta .
$$

Using the result

$$
\int_{0}^{\infty} \frac{\sin (\eta t)}{\eta\left(\xi^{2}+\eta^{2}\right)} d \eta=\frac{\pi\left(1-e^{-\xi t}\right)}{2 \xi^{2}},
$$

we find with the use of (48) that

$$
\int_{0}^{\infty} \frac{\xi}{\xi^{2}+\eta^{2}} A_{1}(\eta) d \eta=\int_{0}^{a} \frac{e^{-\xi t}}{\xi} g(t) d t .
$$

Substitution of (53) into (42) yields the expressions for $B_{1}(\xi), B_{2}(\xi), C_{1}(\xi)$, and $D_{1}(\xi)$ in terms of $g(x)$.

From fraction-free condition (22) from $(28)_{1}$, one can derive

$$
\begin{aligned}
& \int_{0}^{\infty} \xi\left[c_{44}^{\mathrm{I}} A_{1}(\xi) \cos (\xi x)\right. \\
& \left.\quad-\left(\tilde{c}_{44}^{\mathrm{I}} B_{1}(\xi)-\alpha^{\mathrm{I}} C_{1}(\xi)-\beta^{\mathrm{I}} D_{1}(\xi)\right) e^{-\xi x}\right] d \xi=\tau_{0}^{\mathrm{I}} .
\end{aligned}
$$

Substituting (50) and (42) with the use of (53) into (54), we have with the help of known integrals

$$
\begin{gathered}
\frac{2}{\pi} \int_{0}^{\infty} \sin (\xi t) \cos (\xi x) d \xi=\frac{1}{\pi}\left(\frac{1}{t-x}+\frac{1}{t+x}\right), \\
\int_{0}^{\infty} e^{-\xi(t+x)} d \xi=\frac{1}{t+x} ; \quad t+x>0
\end{gathered}
$$

the following singular integral equation with generalized Cauchy kernel for $g(t)$ :

$$
\frac{1}{\pi} \int_{0}^{a}\left(\frac{1}{t-x}+\frac{\lambda}{t+x}\right) g(t) d t=-\frac{\tau_{0}^{\mathrm{I}}}{c_{44}^{\mathrm{I}}} ; \quad 0<x<a,
$$

where 


$$
\begin{aligned}
\lambda= & \left.1-2 \frac{\tilde{c}_{44}^{\mathrm{II}} \Delta+\left(e_{3}^{\mathrm{II}}+e_{3}^{\mathrm{I}}\right) \alpha^{\mathrm{II}}\left(\alpha^{\mathrm{II}}-\alpha^{\mathrm{I}}\right)+\left(e_{1}^{\mathrm{II}}+e_{1}^{\mathrm{I}}\right) \beta^{\mathrm{II}}\left(\beta^{\mathrm{II}}-\beta^{\mathrm{I}}\right)-\left(e_{2}^{\mathrm{II}}+e_{2}^{\mathrm{I}}\right)\left(\alpha^{\mathrm{I}} \beta^{\mathrm{II}}+\beta^{\mathrm{I}} \alpha^{\mathrm{II}}+2 \alpha^{\mathrm{II}} \beta^{\mathrm{II}}\right)}{\left(\tilde{c}_{44}^{\mathrm{I}}+\tilde{c}_{44}^{\mathrm{II}}\right) \Delta+\left(e_{3}^{\mathrm{II}}+e_{3}^{\mathrm{I}}\right)\left(\alpha^{\mathrm{II}}-\alpha^{\mathrm{I}}\right)^{2}+\left(e_{1}^{\mathrm{II}}+e_{1}^{\mathrm{I}}\right)\left(\beta^{\mathrm{II}}-\beta^{\mathrm{I}}\right)^{2}}\right] \\
& \times\left[\frac{\tilde{c}_{44}^{\mathrm{I}}}{c_{44}^{\mathrm{I}}}-\frac{\left(e_{3}^{\mathrm{II}}+e_{3}^{\mathrm{I}}\right) \alpha^{\mathrm{I}}\left(\alpha^{\mathrm{II}}-\alpha^{\mathrm{I}}\right)+\left(e_{1}^{\mathrm{II}}+e_{1}^{\mathrm{I}}\right) \beta^{\mathrm{I}}\left(\beta^{\mathrm{II}}-\beta^{\mathrm{I}}\right)-\left(e_{2}^{\mathrm{II}}+e_{2}^{\mathrm{I}}\right)\left(\alpha^{\mathrm{I}} \beta^{\mathrm{II}}+\beta^{\mathrm{I}} \alpha^{\mathrm{II}}-2 \alpha^{\mathrm{I}} \beta^{\mathrm{I}}\right)}{c_{44}^{\mathrm{I}} \Delta}\right] \\
& +2 \frac{\left(e_{3}^{\mathrm{II}}+e_{3}^{\mathrm{I}}\right) \alpha^{\mathrm{I}} \alpha^{\mathrm{II}}+\left(e_{1}^{\mathrm{II}}+e_{1}^{\mathrm{I}}\right) \beta^{\mathrm{I}} \beta^{\mathrm{II}}-\left(e_{2}^{\mathrm{II}}+e_{2}^{\mathrm{I}}\right)\left(\alpha^{\mathrm{I}} \beta^{\mathrm{II}}+\beta^{\mathrm{I}} \alpha^{\mathrm{II}}\right)}{c_{44}^{\mathrm{I}} \Delta} .
\end{aligned}
$$

For both piezoelectric materials, $\lambda$ is obtained as follows:

$$
\lambda=\frac{\left(c_{44}^{\mathrm{I}}-c_{44}^{\mathrm{II}}\right)\left(\varepsilon_{11}^{\mathrm{I}}+\varepsilon_{11}^{\mathrm{II}}\right)+\left(e_{15}^{\mathrm{I}}\right)^{2}-\left(e_{15}^{\mathrm{II}}\right)^{2}+2 e_{15}^{\mathrm{I}}\left(e_{15}^{\mathrm{II}}-e_{15}^{\mathrm{I}}\left(c_{44}^{\mathrm{II}} / c_{44}^{\mathrm{I}}\right)\right)}{\left(c_{44}^{\mathrm{I}}+c_{44}^{\mathrm{II}}\right)\left(\varepsilon_{11}^{\mathrm{I}}+\varepsilon_{11}^{\mathrm{II}}\right)+\left(e_{15}^{\mathrm{I}}+e_{15}^{\mathrm{II}}\right)^{2}} .
$$

The value of $\lambda$ for both piezomagnetic materials is obtained from formula (58) if we replace $\varepsilon_{11}$ by $\mu_{11}$ and $e_{15}$ by $q_{15}$. It is noted that, in a usual integral equation with Cauchy kernel, other kernels except Cauchy kernel are continuous over the entire interval involved. In addition to the singularity of the Cauchy kernel terms $1 /(t-x)$ as $t \rightarrow x$ in (56), the other term $\lambda /(t+x)$ is also unbonded as $t, x \rightarrow$ 0 simultaneously. Particularly for two elastic dielectric, meaning $e_{15}=0$, and diamagnetic, meaning $q_{15}=0$, elastic field and electric field, and elastic field and magnetic field are not coupled as well as when $d_{11}=0$, the electromagnetic field does not occur. In this case, $\lambda$ reduces to

$$
\lambda=\frac{c_{44}^{\mathrm{I}}-c_{44}^{\mathrm{II}}}{c_{44}^{\mathrm{I}}+c_{44}^{\mathrm{II}}}
$$

Then the integral equation is simplified to

$$
\frac{1}{\pi} \int_{0}^{a}\left(\frac{1}{t-x}+\frac{\lambda}{t+x}\right) g(t) d t=-\frac{\tau_{0}}{c_{44}} .
$$

This equation is equivalent to that derived by Cook and Erdogan [6] and Erdogan and Cook [7], who were apparently the first to publish the solution of an antiplane shear crack terminating at the interface of two joined purely elastic media.

\section{Magnetoelectroelastic Field}

4.1. Solution of the Singular Integral Equation. Based on the result derived by Bueckner [10], the desired solution for $g(t)$ of (56) subjected to (48) can be obtained as follows:

$$
g(x)=\frac{\tau_{0}^{\mathrm{I}}}{2 c_{44}^{\mathrm{I}} \sin (\pi \alpha / 2)}
$$

$$
\begin{aligned}
& \times\left[\left(\frac{x}{a+\sqrt{a^{2}-x^{2}}}\right)^{\alpha}\left(\frac{\alpha a}{\sqrt{a^{2}-x^{2}}}+1\right)\right. \\
& \left.\quad+\left(\frac{x}{a+\sqrt{a^{2}-x^{2}}}\right)^{-\alpha}\left(\frac{\alpha a}{\sqrt{a^{2}-x^{2}}}-1\right)\right]
\end{aligned}
$$

for $0<x<a$ with

$$
\cos (\pi \alpha)=-\lambda
$$

where $0<\alpha<1$.

Once $g(t)$ is determined the crack tearing displacement can be obtained by the following integrations:

$$
\begin{aligned}
& w^{\mathrm{I}}\left(x, 0^{+}\right)= \int_{0}^{x} g(x) d x \\
&=-\frac{\tau_{0}^{\mathrm{I} x}}{2 c_{44}^{\mathrm{I}} \sin (\pi \alpha / 2)} \\
& \times\left[\left(\frac{x}{a+\sqrt{a^{2}-x^{2}}}\right)^{\alpha}-\left(\frac{x}{a+\sqrt{a^{2}-x^{2}}}\right)^{-\alpha}\right] . \\
& 0 \leq x \leq a
\end{aligned}
$$

4.2. Crack Tearing Displacement. Expanding the expression (63) near the crack tips yields the asymptotic crack tearing displacement as

$$
\begin{gathered}
w^{\mathrm{I}}(x, 0)=\frac{\tau_{0}^{\mathrm{I}}}{c_{44}^{\mathrm{I}}} \frac{\alpha}{\sin (\pi \alpha / 2)} \sqrt{2 a(a-x)}+O(r) ; \quad r=a-x \approx 0 \\
w^{\mathrm{I}}(x, 0)=\frac{\tau_{0}^{\mathrm{I}} a^{\alpha}}{2 c_{44}^{\mathrm{I}} \sin (\pi \alpha / 2)} x^{1-\alpha}+O(r) ; \quad r=x \approx 0
\end{gathered}
$$

at the right and left crack tip. 


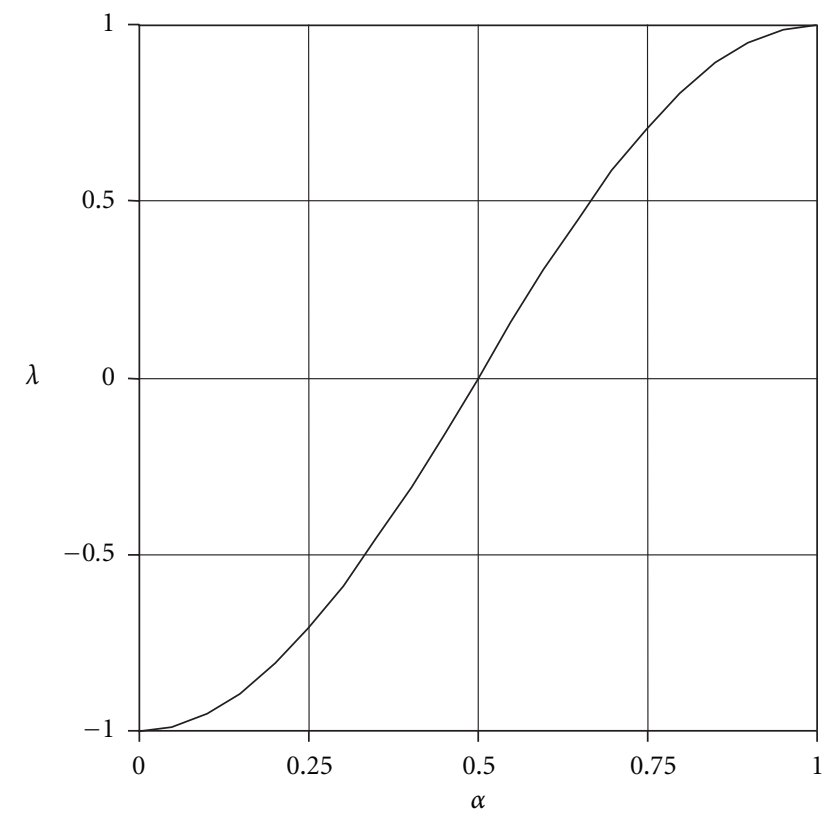

Figure 2: The curve $\lambda=-\cos (\pi \alpha) ; \lambda$ is the bi-material parameter, and $\alpha$ is singularity order parameter.

Here $O(r)$ denotes the infinitesimal terms compared to $r$, $r$ being the distance from the crack tip. Only for $\alpha=1 / 2$ the behaviours of the crack tearing displacement for both tips are the same.

4.3. Asymptotic Crack-Tip Field. Antiplane shear crack and in-plane electric displacement and magnetic induction may be deduced by evaluating the following integrals:

$$
\begin{gathered}
\tau_{y z}^{\mathrm{I}}(x, 0)=\frac{1}{\pi} c_{44}^{\mathrm{I}} \int_{0}^{a}\left(\frac{1}{t-x}+\frac{\lambda}{t+x}\right) g(t) d t+\tau_{0}^{\mathrm{I}}, \\
D_{y}^{\mathrm{I}}(x, 0)=\frac{1}{\pi} e_{15}^{\mathrm{I}} \int_{0}^{a}\left(\frac{1}{t-x}+\frac{1-2 \lambda_{D}}{1+t}\right) g(t) d t+D^{\mathrm{I}}, \\
B_{y}^{\mathrm{I}}(x, 0)=\frac{1}{\pi} q_{15}^{\mathrm{I}} \int_{0}^{a}\left(\frac{1}{t-x}+\frac{1-2 \lambda_{B}}{1+t}\right) g(t) d t+B^{\mathrm{I}},
\end{gathered}
$$

for $x>a$ and

$$
\begin{gathered}
\tau_{y z}^{\mathrm{II}}(x, 0)=\frac{1-\lambda}{\pi} c_{44}^{\mathrm{I}} \int_{0}^{a} \frac{g(t)}{t-x} d t+\tau_{0}^{\mathrm{II}}, \\
D_{y}^{\mathrm{II}}(x, 0)=\frac{2 \lambda_{D}}{\pi} e_{15}^{\mathrm{I}} \int_{0}^{a} \frac{g(t)}{t-x} d t+D^{\mathrm{II}}, \\
B_{y}^{\mathrm{II}}(x, 0)=\frac{2 \lambda_{B}}{\pi} q_{15}^{\mathrm{I}} \int_{0}^{a} \frac{g(t)}{t-x} d t+B^{\mathrm{II}}
\end{gathered}
$$

for $x<0$, where $1-\lambda$ is defined by (57) and

$$
\begin{aligned}
& e_{15}^{\mathrm{I}} \lambda_{D}=\left\{\Delta \left[\left(\tilde{c}_{44}^{\mathrm{II}} \alpha^{\mathrm{I}}+\tilde{c}_{44}^{\mathrm{I}} \alpha^{\mathrm{II}}\right)\left(e_{3}^{\mathrm{II}}+e_{3}^{\mathrm{I}}\right)\right.\right. \\
& \left.-\left(\widetilde{c}_{44}^{\mathrm{II}} \beta^{\mathrm{I}}+\widetilde{c}_{44}^{\mathrm{I}} \beta^{\mathrm{II}}\right)\left(e_{2}^{\mathrm{II}}+e_{2}^{\mathrm{I}}\right)\right]+\left(e_{3}^{\mathrm{II}}+e_{3}^{\mathrm{I}}\right) \\
& \times\left(e_{2}^{\mathrm{II}}+e_{2}^{\mathrm{I}}\right)\left(\alpha^{\mathrm{II}}-\alpha^{\mathrm{I}}\right)\left(\alpha^{\mathrm{I}} \beta^{\mathrm{II}}+\beta^{\mathrm{I}} \alpha^{\mathrm{II}}+2 \alpha^{\mathrm{II}} \beta^{\mathrm{II}}\right) \\
& +\left(e_{3}^{\mathrm{II}}+e_{3}^{\mathrm{I}}\right)\left(e_{1}^{\mathrm{II}}+e_{1}^{\mathrm{I}}\right)\left(\beta^{\mathrm{II}}-\beta^{\mathrm{I}}\right)\left(\alpha^{\mathrm{I}} \beta^{\mathrm{II}}-\beta^{\mathrm{I}} \alpha^{\mathrm{II}}\right) \\
& \left.+\left(e_{2}^{\mathrm{II}}+e_{2}^{\mathrm{I}}\right)^{2}\left(\beta^{\mathrm{II}}-\beta^{\mathrm{I}}\right)\left(\alpha^{\mathrm{I}} \beta^{\mathrm{II}}+\beta^{\mathrm{I}} \alpha^{\mathrm{II}}+2 \alpha^{\mathrm{II}} \beta^{\mathrm{II}}\right)\right\} \\
& \times\left\{\Delta \left[\left(\tilde{c}_{44}^{\mathrm{I}}+\tilde{c}_{44}^{\mathrm{II}}\right) \Delta+\left(e_{3}^{\mathrm{II}}+e_{3}^{\mathrm{I}}\right)\left(\alpha^{\mathrm{II}}-\alpha^{\mathrm{I}}\right)^{2}\right.\right. \\
& \left.\left.+\left(e_{1}^{\mathrm{II}}+e_{1}^{\mathrm{I}}\right)\left(\beta^{\mathrm{II}}-\beta^{\mathrm{I}}\right)^{2}\right]\right\}^{-1}, \\
& q_{15}^{\mathrm{I}} \lambda_{B}=\left\{\Delta \left[\left(\widetilde{c}_{44}^{\mathrm{II}} \beta^{\mathrm{I}}+\widetilde{c}_{44}^{\mathrm{I}} \beta^{\mathrm{II}}\right)\left(e_{1}^{\mathrm{II}}+e_{1}^{\mathrm{I}}\right)\right.\right. \\
& \left.-\left(\tilde{c}_{44}^{\mathrm{II}} \alpha^{\mathrm{I}}+\tilde{c}_{44}^{\mathrm{I}} \alpha^{\mathrm{II}}\right)\left(e_{2}^{\mathrm{II}}+e_{2}^{\mathrm{I}}\right)\right]-\left(e_{1}^{\mathrm{II}}+e_{1}^{\mathrm{I}}\right) \\
& \times\left(e_{2}^{\mathrm{II}}+e_{2}^{\mathrm{I}}\right)\left(\beta^{\mathrm{II}}-\beta^{\mathrm{I}}\right)\left(\alpha^{\mathrm{I}} \beta^{\mathrm{II}}+\beta^{\mathrm{I}} \alpha^{\mathrm{II}}+2 \alpha^{\mathrm{II}} \beta^{\mathrm{II}}\right) \\
& -\left(e_{2}^{\mathrm{II}}+e_{2}^{\mathrm{I}}\right)\left(e_{1}^{\mathrm{II}}+e_{1}^{\mathrm{I}}\right)\left(\beta^{\mathrm{II}}-\beta^{\mathrm{I}}\right)\left(\alpha^{\mathrm{I}} \beta^{\mathrm{II}}-\beta^{\mathrm{I}} \alpha^{\mathrm{II}}\right) \\
& \left.-\left(e_{2}^{\mathrm{II}}+e_{2}^{\mathrm{I}}\right)^{2}\left(\alpha^{\mathrm{II}}-\alpha^{\mathrm{I}}\right)\left(\alpha^{\mathrm{I}} \beta^{\mathrm{II}}+\beta^{\mathrm{I}} \alpha^{\mathrm{II}}+2 \alpha^{\mathrm{II}} \beta^{\mathrm{II}}\right)\right\} \\
& \times\left\{\Delta \left[\left(\widetilde{c}_{44}^{\mathrm{I}}+\widetilde{c}_{44}^{\mathrm{II}}\right) \Delta+\left(e_{3}^{\mathrm{II}}+e_{3}^{\mathrm{I}}\right)\left(\alpha^{\mathrm{II}}-\alpha^{\mathrm{I}}\right)^{2}\right.\right. \\
& \left.\left.+\left(e_{1}^{\mathrm{II}}+e_{1}^{\mathrm{I}}\right)\left(\beta^{\mathrm{II}}-\beta^{\mathrm{I}}\right)^{2}\right]\right\}^{-1} .
\end{aligned}
$$
give

For both piezoelectric or piezomagnetic materials, (67)

$$
\begin{gathered}
e_{15}^{\mathrm{I}} \lambda_{D}=\frac{e_{15}^{\mathrm{I}}\left(c_{44}^{\mathrm{II}} \varepsilon_{11}^{\mathrm{II}}+\left(e_{15}^{\mathrm{II}}\right)^{2}\right)+e_{15}^{\mathrm{II}}\left(c_{44}^{\mathrm{I}} \varepsilon_{11}^{\mathrm{I}}+\left(e_{15}^{\mathrm{I}}\right)^{2}\right)}{\left(c_{44}^{\mathrm{I}}+c_{44}^{\mathrm{II}}\right)\left(\varepsilon_{11}^{\mathrm{I}}+\varepsilon_{11}^{\mathrm{II}}\right)+\left(e_{15}^{\mathrm{I}}+e_{15}^{\mathrm{II}}\right)^{2}}, \\
q_{15}^{\mathrm{I}} \lambda_{B}=0
\end{gathered}
$$

or

$$
e_{15}^{\mathrm{I}} \lambda_{D}=0,
$$

$$
q_{15}^{\mathrm{I}} \lambda_{B}=\frac{q_{15}^{\mathrm{I}}\left(c_{44}^{\mathrm{II}} \mu_{11}^{\mathrm{II}}+\left(q_{15}^{\mathrm{II}}\right)^{2}\right)+q_{15}^{\mathrm{II}}\left(c_{44}^{\mathrm{I}} \mu_{11}^{\mathrm{I}}+\left(q_{15}^{\mathrm{I}}\right)^{2}\right)}{\left(c_{44}^{\mathrm{I}}+c_{44}^{\mathrm{II}}\right)\left(\mu_{11}^{\mathrm{I}}+\mu_{11}^{\mathrm{II}}\right)+\left(q_{15}^{\mathrm{I}}+q_{15}^{\mathrm{II}}\right)^{2}} .
$$

The analytical expressions for physical quantities may be obtained substituting the solution (61) into (65) and (66). We omit full solution and pay our attentions to the asymptotic crack-tip field. This is very interest from the view point of fracture mechanics. From (61), one can write out 
the singular behaviour of the function $g(x)$ near the point $x=0$ and $x=a$ by the following asymptotic expressions:

$$
\begin{aligned}
& g(x)=-\frac{\tau_{0}^{\mathrm{I}}}{2 c_{44}^{\mathrm{I}}} \frac{\alpha}{\sin (\pi \alpha / 2)} \sqrt{\frac{2 a}{a-x}}+O(1) ; \quad x \approx a-0, \\
& g(x)=-\frac{\tau_{0}^{\mathrm{I}}}{2 c_{44}^{\mathrm{I}}} \frac{\alpha-1}{\sin (\pi \alpha / 2)}\left[\frac{2(a-x)}{x}\right]^{\alpha}+O(1) ; \quad x \approx 0+0,
\end{aligned}
$$

where $O(1)$ stands for nonsingular terms.

Now we define the intensity factor at the right crack tip in the homogeneous solid and the left crack tip at the interface of a bimedium as

$$
\begin{aligned}
K_{\mathrm{hom}}^{q} & =\lim _{x \rightarrow a^{+}} \sqrt{2 \pi(x-a)} q^{\mathrm{I}}\left(x, 0^{+}\right), \\
K_{\mathrm{int}}^{q} & =\lim _{x \rightarrow 0^{-}}(-2 \pi x)^{\alpha} q^{\mathrm{II}}\left(x, 0^{+}\right),
\end{aligned}
$$

respectively, where $q$ stands for one of $\tau_{y z}, \gamma_{y z}, D_{y}, B_{y}, E_{y}$, and $H_{y}$.

4.3.1. Magnetoelectroelastic Field near the Crack Tip in the Homogeneous PEMO-Elastic Ceramics. Using the integral

$$
\frac{1}{\pi} \int_{0}^{a} \frac{1}{(t-x) \sqrt{a-t}} d t=-\frac{2}{\pi \sqrt{x-a}} \tan ^{-1} \sqrt{\frac{a}{x-a}}, \quad x>a,
$$

we obtain from $(65)_{1}$ that

$$
\tau_{y z}^{\mathrm{I}}(x, 0)=\frac{1}{\pi} c_{44}^{\mathrm{I}} \int_{0}^{a} \frac{g(t)}{t-x} d t+O(1)=\frac{K_{\mathrm{hom}}^{\tau}}{\sqrt{2 \pi(x-a)}},
$$

where

$$
K_{\text {hom }}^{\tau}=\frac{\alpha}{\sin (\pi \alpha / 2)} \tau_{o}^{\mathrm{I}} \sqrt{\pi a}
$$

the stress intensity factor at the right crack tip. Other field intensity factors are related to $K_{\text {hom }}^{\tau}$ as follows:

$$
\begin{gathered}
K_{\text {hom }}^{\gamma}=\frac{1}{c_{44}^{I}} K_{\text {hom }}^{\tau}, \quad K_{\text {hom }}^{D}=\frac{e_{15}^{I}}{c_{44}^{I}} K_{\text {hom }}^{\tau}, \quad K_{\text {hom }}^{B}=\frac{q_{15}^{\mathrm{I}}}{c_{44}^{I}} K_{\text {hom }}^{\tau}, \\
K_{\text {hom }}^{\phi}=K_{\text {hom }}^{\psi}=K_{\text {hom }}^{E}=K_{\text {hom }}^{H}=0 .
\end{gathered}
$$

For the crack tip in homogeneous PEMO-elastic medium the elastic, electric, and magnetic fields still exhibit an inverse square-root singularity at the crack tip. Application of electric and magnetic fields does not alter the stress intensity factors. The stress intensity factor depends on the material properties of two PEMO-elastic ceramics involved since it is governed by (75) and $\alpha$ by (62). The intensity factors $K_{\text {hom }}^{\gamma}, K_{\text {hom }}^{D}$, and $K_{\text {hom }}^{B}$ are related to $K_{\text {hom }}^{\tau}$ and also depend on the material properties, as shown in (76).
4.3.2. Magnetoelectroelastic Field near the Crack Tip at the Interface. Using the known result [11],

$$
\frac{1}{\pi} \int_{0}^{a} \frac{1}{(t-x)}\left(\frac{a-t}{t}\right)^{\alpha} d t=\frac{1}{\sin (\pi \alpha)}\left[\left(\frac{x-a}{x}\right)^{\alpha}-1\right], \quad x<0
$$

putting (71) into (66) and using (77), we obtain the asymptotic expressions for the antiplane shear stress and in-plane electric displacement and magnetic induction, as well as elastic strain, electric and magnetic field, ahead on the left crack tip at the interface as follows:

$$
\begin{aligned}
{\left[K_{\text {int }}^{\tau} ; K_{\text {int }}^{D} ; K_{\text {int }}^{B} ; K_{\text {int }}^{\gamma} ; K_{\text {int }}^{E} ; K_{\text {int }}^{H}\right] } \\
=\frac{\sqrt{2}(1-\alpha)}{(1+\lambda) \sqrt{1-\lambda}} \frac{\tau_{0}^{\mathrm{I}}}{c_{44}^{\mathrm{I}}}(4 \pi a)^{\alpha} \\
\quad \times\left[c_{44}^{\mathrm{I}} \frac{1-\lambda}{2} ; e_{15}^{\mathrm{I}} \lambda_{D} ; q_{15}^{\mathrm{I}} \lambda_{B} ; \lambda_{\gamma} ; \lambda_{E} ; \lambda_{H}\right],
\end{aligned}
$$

where the identity is used as follows:

$$
\sin (\pi \alpha) \sin \left(\frac{\pi \alpha}{2}\right)=(1+\lambda) \sqrt{\frac{1-\lambda}{2}}
$$

and where

$$
\begin{aligned}
& \lambda_{\gamma}=\left[\tilde{c}_{44}^{\mathrm{I}} \Delta-\left(e_{3}^{\mathrm{II}}+e_{3}^{\mathrm{I}}\right) \alpha^{\mathrm{I}}\left(\alpha^{\mathrm{II}}-\alpha^{\mathrm{I}}\right)-\left(e_{1}^{\mathrm{II}}+e_{1}^{\mathrm{I}}\right) \beta^{\mathrm{I}}\left(\beta^{\mathrm{II}}-\beta^{\mathrm{I}}\right)\right. \\
& \left.+\left(e_{2}^{\mathrm{II}}+e_{2}^{\mathrm{I}}\right)\left(\alpha^{\mathrm{I}} \beta^{\mathrm{II}}+\beta^{\mathrm{I}} \alpha^{\mathrm{II}}+2 \alpha^{\mathrm{II}} \beta^{\mathrm{II}}\right)\right] \\
& \times\left[\left(\tilde{c}_{44}^{\mathrm{I}}+\tilde{c}_{44}^{\mathrm{II}}\right) \Delta+\left(e_{3}^{\mathrm{II}}+e_{3}^{\mathrm{I}}\right)\left(\alpha^{\mathrm{II}}-\alpha^{\mathrm{I}}\right)^{2}\right. \\
& \left.+\left(e_{1}^{\mathrm{II}}+e_{1}^{\mathrm{I}}\right)\left(\beta^{\mathrm{II}}-\beta^{\mathrm{I}}\right)^{2}\right]^{-1} \\
& \lambda_{E}=\left\{\lambda _ { \gamma } \left[\alpha^{\mathrm{I}} \Delta+\left(\alpha^{\mathrm{II}}-\alpha^{\mathrm{I}}\right)\left(e_{1}^{\mathrm{I}}\left(e_{3}^{\mathrm{II}}+e_{3}^{\mathrm{I}}\right)-e_{2}^{\mathrm{I}}\left(e_{2}^{\mathrm{II}}+e_{2}^{\mathrm{I}}\right)\right)\right.\right. \\
& \left.-\left(\beta^{\mathrm{II}}-\beta^{\mathrm{I}}\right)\left(e_{1}^{\mathrm{I}} e_{2}^{\mathrm{II}}-e_{2}^{\mathrm{I}} e_{1}^{\mathrm{II}}\right)\right] \\
& -\alpha^{\mathrm{II}}\left(e_{1}^{\mathrm{I}}\left(e_{3}^{\mathrm{II}}+e_{3}^{\mathrm{I}}\right)-e_{2}^{\mathrm{I}}\left(e_{2}^{\mathrm{II}}+e_{2}^{\mathrm{I}}\right)\right) \\
& \left.+\beta^{\mathrm{II}}\left(e_{1}^{\mathrm{I}} e_{2}^{\mathrm{II}}-e_{2}^{\mathrm{I}} e_{1}^{\mathrm{II}}\right)\right\} \Delta^{-1} \\
& \lambda_{H}=\left\{\lambda _ { \gamma } \left[\beta^{\mathrm{I}} \Delta+\left(\beta^{\mathrm{II}}-\beta^{\mathrm{I}}\right)\left(e_{3}^{\mathrm{I}}\left(e_{1}^{\mathrm{II}}+e_{1}^{\mathrm{I}}\right)-e_{2}^{\mathrm{I}}\left(e_{2}^{\mathrm{II}}+e_{2}^{\mathrm{I}}\right)\right)\right.\right. \\
& \left.-\left(\alpha^{\mathrm{II}}-\alpha^{\mathrm{I}}\right)\left(e_{2}^{\mathrm{I}} e_{3}^{\mathrm{II}}-e_{3}^{\mathrm{I}} e_{2}^{\mathrm{II}}\right)\right] \\
& -\beta^{\mathrm{II}}\left(e_{2}^{\mathrm{I}}\left(e_{2}^{\mathrm{II}}+e_{2}^{\mathrm{I}}\right)-e_{3}^{\mathrm{I}}\left(e_{1}^{\mathrm{II}}+e_{1}^{\mathrm{I}}\right)\right) \\
& \left.+\alpha^{\mathrm{II}}\left(e_{2}^{\mathrm{I}} e_{3}^{\mathrm{II}}-e_{3}^{\mathrm{I}} e_{2}^{\mathrm{II}}\right)\right\} \Delta^{-1}
\end{aligned}
$$


for PEMO-elastic bimaterial and

$$
\begin{gathered}
\lambda_{\gamma}=\frac{c_{44}^{\mathrm{I}}\left(\varepsilon_{11}^{\mathrm{I}}+\varepsilon_{11}^{\mathrm{II}}\right)+e_{15}^{\mathrm{I}}\left(e_{15}^{\mathrm{I}}+e_{15}^{\mathrm{II}}\right)}{\left(c_{44}^{\mathrm{I}}+c_{44}^{\mathrm{II}}\right)\left(\varepsilon_{11}^{\mathrm{I}}+\varepsilon_{11}^{\mathrm{II}}\right)+\left(e_{15}^{\mathrm{I}}+e_{15}^{\mathrm{II}}\right)^{2}}, \\
\lambda_{E}=\frac{c_{44}^{\mathrm{II}} e_{15}^{\mathrm{I}}-c_{44}^{\mathrm{I}} e_{15}^{\mathrm{II}}}{\left(c_{44}^{\mathrm{I}}+c_{44}^{\mathrm{II}}\right)\left(\varepsilon_{11}^{\mathrm{I}}+\varepsilon_{11}^{\mathrm{II}}\right)+\left(e_{15}^{\mathrm{I}}+e_{15}^{\mathrm{II}}\right)^{2}}, \\
\lambda_{H}=0
\end{gathered}
$$

for piezoelectric bi-material and

$$
\begin{gathered}
\lambda_{\gamma}=\frac{c_{44}^{\mathrm{I}}\left(\mu_{11}^{\mathrm{I}}+\mu_{11}^{\mathrm{II}}\right)+q_{15}^{\mathrm{I}}\left(q_{15}^{\mathrm{I}}+q_{15}^{\mathrm{II}}\right)}{\left(c_{44}^{\mathrm{I}}+c_{44}^{\mathrm{II}}\right)\left(\mu_{11}^{\mathrm{I}}+\mu_{11}^{\mathrm{II}}\right)+\left(q_{15}^{\mathrm{I}}+q_{15}^{\mathrm{II}}\right)^{2}}, \\
\lambda_{E}=0, \\
\lambda_{H}=\frac{c_{44}^{\mathrm{II}} q_{15}^{\mathrm{I}}-c_{44}^{\mathrm{I}} q_{15}^{\mathrm{II}}}{\left(c_{44}^{\mathrm{I}}+c_{44}^{\mathrm{II}}\right)\left(\mu_{11}^{\mathrm{I}}+\mu_{11}^{\mathrm{II}}\right)+\left(q_{15}^{\mathrm{I}}+q_{15}^{\mathrm{II}}\right)^{2}}
\end{gathered}
$$

for piezomagnetic bi-material.

Note that for piezoelectric bi-material, we have

$$
\lambda_{\tau}=\frac{1-\lambda}{2}=\frac{c_{44}^{\mathrm{I}}\left(\varepsilon_{11}^{\mathrm{I}}+\varepsilon_{11}^{\mathrm{II}}\right)+\left(e_{15}^{\mathrm{II}}\right)^{2}+\left(e_{15}^{\mathrm{I}}\right)^{2}\left(c_{44}^{\mathrm{II}} / c_{44}^{\mathrm{I}}\right)}{\left(c_{44}^{\mathrm{I}}+c_{44}^{\mathrm{II}}\right)\left(\varepsilon_{11}^{\mathrm{I}}+\varepsilon_{11}^{\mathrm{II}}\right)+\left(e_{15}^{\mathrm{I}}+e_{15}^{\mathrm{II}}\right)^{2}}
$$

The material parameters for piezoelectric ceramics coincide, in general, with the ones derived by Li and Wang [9]. But in $\lambda$, defined exactly by (58), the fourth term in numerator of (58) is omitted in (46) of Li and Wang paper. In consequence, the conclusions in Table 2 of $\mathrm{Li}$ and Wang paper that $\lambda$ vanishes also in the case of ceramics poled in opposite direction are incorrect. The formula (58) shows that only for two bonded piezoelectric ceramics with $c_{44}$ unchanged poled in the same direction (not opposite) the field singularity at the interface crack tip maintains the inverse square root singularity, since in this case is $\lambda=0$ and $\alpha=1 / 2$. The parameter $\lambda_{E}$ in this paper has opposite sign to that presented by $\mathrm{Li}$ and Wang. This gives that for $\rho_{c}>1\left(c_{44}^{\mathrm{II}}>c_{44}^{\mathrm{I}}\right)$ meaning that piezoelectric ceramic II is more stiffer that piezoelectric ceramic I $\left(e_{15}^{\mathrm{II}}=e_{15}^{\mathrm{I}}\right)$, in this case $\lambda_{E}>0$, so stands also $K_{\text {int }}^{E}>0$, and $K_{\text {int }}^{E}$ increases with $\rho_{c}$. Also it is seen that $K_{\text {int }}^{E}$ decreases with the ratio $\rho_{e}$ of $e_{15}^{\mathrm{II}}$ to $e_{15}^{\mathrm{I}}$. In the paper Li and Wang [9], the conclusions, associated with $K_{\text {int }}^{E}$, are inverse. The presented conclusions are consistent with physical consideration. The field intensity factors must satisfy the constitutive equations

$$
K^{\tau}=c_{44}^{\mathrm{II}} K^{\gamma}-e_{15}^{\mathrm{II}} K^{E}, \quad K^{D}=e_{15}^{\mathrm{II}} K^{\gamma}+\varepsilon_{11}^{\mathrm{II}} K^{E},
$$

or material parameters must satisfy the equivalent equations

$$
c_{44}^{\mathrm{I}} \lambda_{\tau}=c_{44}^{\mathrm{II}} \lambda_{\gamma}-e_{15}^{\mathrm{II}} \lambda_{E}, \quad e_{15}^{\mathrm{I}} \lambda_{D}=e_{15}^{\mathrm{II}} \lambda_{\gamma}+\varepsilon_{11}^{\mathrm{II}} \lambda_{E} .
$$

It is easily verified that both constitutive relations (85) are satisfied by the coefficients defined by (68), (81), and (83). In general, for Magnetoelectroelastic ceramic, the field intensity factors must satisfy the constitutive equations

$$
\left[\begin{array}{c}
K_{\text {int }}^{\tau} \\
K_{\text {int }}^{\phi} \\
K_{\text {int }}^{\psi}
\end{array}\right]=\left[\begin{array}{ccc}
\tilde{c}_{44} & -\alpha & -\beta \\
\alpha & e_{1} & e_{2} \\
\beta & e_{2} & e_{3}
\end{array}\right]\left[\begin{array}{c}
K^{w} \\
K^{D} \\
K^{B}
\end{array}\right],
$$

as shown in (14). Of course, we have $K_{\text {int }}^{\phi}=-K_{\text {int }}^{E}$ and $K_{\text {int }}^{\psi}=$ $-K_{\text {int }}^{H}$.

4.4. The Energy Release Rate. For magnetoelectrically permeable crack, the energy release rates are very important to evaluate the behaviours of crack tips. In accordance with the definition of the energy release rate proposed by [12] (the virtual crack closure integral), the energy release rate can finally be derived as

$$
\begin{aligned}
G & =\frac{1}{2 c_{44}^{\mathrm{I}}}\left[\left(K_{\text {hom }}^{\tau}\right)^{2}+\left(\frac{\pi a}{2}\right)^{1-2 \alpha}\left(K_{\text {int }}^{\tau}\right)^{2}\right] \\
& =G_{\text {hom }} \frac{1}{2}\left[\left(k_{\text {hom }}^{\tau}\right)^{2}+\left(k_{\text {int }}^{\tau}\right)^{2}\right],
\end{aligned}
$$

where

$$
\begin{aligned}
& G_{\text {hom }}=\frac{\pi a\left(\tau_{0}^{\mathrm{I}}\right)^{2}}{2 c_{44}^{\mathrm{I}}}, \\
& k_{\text {hom }}^{\tau}=\frac{K_{\text {hom }}^{\tau}}{\tau_{0}^{\mathrm{I}} \sqrt{\pi a / 2}}, \\
& k_{\text {int }}^{\tau}=\frac{K_{\text {int }}^{\tau}}{\tau_{0}^{\mathrm{I}}(\pi a / 2)^{\alpha}}
\end{aligned}
$$

are the energy release rate for homogeneous material (no bimaterial) and normalized stress intensity factors at right and left crack tip. One interesting observation from equation (87) is that though the energy release rate, $G$, is independent on the applied electric-magnetic load, it is affected by electricmagnetic properties of two constituents of the bi-material media.

4.5. Electric Displacement and Magnetic Induction inside the Crack. Since the medium inside the crack (usually air or vacuum) allows some penetrations of the some electric and magnetic fields, these fields may not be zero. Suppose that the normal components of the electric displacement and magnetic induction inside the crack are $d_{0}$ and $b_{0}$, respectively. Then from permeable crack boundary conditions (18) and solutions (78), it follows that the quantities $d_{0}$ and $b_{0}$ are as follows:

$$
d_{0}=\left\{\begin{array}{l}
\frac{D_{0}^{\mathrm{I}}-\frac{e_{15}^{\mathrm{I}} \tau_{0}^{\mathrm{I}}}{c_{44}^{\mathrm{I}}} \frac{2 \lambda_{D}}{1-\lambda},}{c_{44}^{\mathrm{I}}}\left(1-\frac{2 \lambda_{D}}{1-\lambda}\right)+\left(\varepsilon_{11}^{\mathrm{I}}+\frac{\left(e_{15}^{\mathrm{I}}\right)^{2}}{c_{44}^{\mathrm{I}}}\right) E_{0}^{\mathrm{I}} \\
\quad+\left(d_{11}^{\mathrm{I}}+\frac{e_{15}^{\mathrm{I}} q_{15}^{\mathrm{I}}}{c_{44}^{\mathrm{I}}}\right) H_{0}^{\mathrm{I}},
\end{array}\right.
$$

case I

case II 


$$
b_{0}=\left\{\begin{array}{cc}
B_{0}^{\mathrm{I}}-\frac{q_{15}^{\mathrm{I}} \tau_{0}^{\mathrm{I}}}{c_{44}^{\mathrm{I}}} \frac{2 \lambda_{B}}{1-\lambda}, & \text { case I } \\
\frac{q_{15}^{\mathrm{I}} \tau_{0}^{\mathrm{I}}}{c_{44}^{\mathrm{I}}}\left(1-\frac{2 \lambda_{B}}{1-\lambda}\right)+\left(\mu_{11}^{\mathrm{I}}+\frac{\left(q_{15}^{\mathrm{I}}\right)^{2}}{c_{44}^{\mathrm{I}}}\right) H_{0}^{\mathrm{I}} & \\
+\left(d_{11}^{\mathrm{I}}+\frac{e_{15}^{\mathrm{I}} q_{15}^{\mathrm{I}}}{c_{44}^{\mathrm{I}}}\right) E_{0}^{\mathrm{I}} . & \text { case II }
\end{array}\right.
$$

Then, using (21), we obtain that

$$
\begin{aligned}
& D^{\mathrm{I}}-d_{0}=\frac{e_{15}^{\mathrm{I}} \tau_{0}^{\mathrm{I}}}{c_{44}^{\mathrm{I}}} \frac{2 \lambda_{D}}{1-\lambda}, \\
& B^{\mathrm{I}}-b_{0}=\frac{q_{15}^{\mathrm{I}} \tau_{0}^{\mathrm{I}}}{c_{44}^{\mathrm{I}}} \frac{2 \lambda_{B}}{1-\lambda}
\end{aligned}
$$

in both cases of loading conditions.

The electric displacement and magnetic induction intensity factors are proportional to $D_{0}^{\mathrm{I}}-d_{0}$ and $B_{0}^{\mathrm{I}}-b_{0}$, respectively [13], and the following relations hold

$$
\begin{gathered}
K_{\text {int }}^{D}=K_{\text {int }}^{\tau} \frac{e_{15}^{\mathrm{I}}}{c_{44}^{\mathrm{I}}} \frac{2 \lambda_{D}}{1-\lambda}, \\
K_{\text {int }}^{B}=K_{\text {int }}^{\tau} \frac{q_{15}^{\mathrm{I}}}{c_{44}^{\mathrm{I}}} \frac{2 \lambda_{B}}{1-\lambda}
\end{gathered}
$$

which are in agreement with the solutions (78). For piezoelectric bi-materials or piezomagnetic bi-materials we have, for instance,

$$
\begin{gathered}
K_{\text {int }}^{D}=K_{\text {int }}^{\tau} \frac{c_{44}^{\mathrm{II}} \varepsilon_{11}^{\mathrm{II}} e_{15}^{\mathrm{I}}+c_{44}^{\mathrm{I}} \varepsilon_{11}^{\mathrm{I}} e_{15}^{\mathrm{II}}+e_{15}^{\mathrm{I}} e_{15}^{\mathrm{II}}\left(e_{15}^{\mathrm{I}}+e_{15}^{\mathrm{II}}\right)}{c_{44}^{\mathrm{II}} c_{44}^{\mathrm{I}}\left(\varepsilon_{11}^{\mathrm{I}}+\varepsilon_{11}^{\mathrm{II}}\right)+c_{44}^{\mathrm{I}}\left(e_{15}^{\mathrm{II}}\right)^{2}+c_{44}^{\mathrm{II}}\left(e_{15}^{\mathrm{I}}\right)^{2}}, \\
K_{\mathrm{int}}^{B}=K_{\mathrm{int}}^{\tau} \frac{c_{44}^{\mathrm{II}} \mu_{11}^{\mathrm{II}} q_{15}^{\mathrm{I}}+c_{44}^{\mathrm{I}} \mu_{11}^{\mathrm{I}} q_{15}^{\mathrm{II}}+q_{15}^{\mathrm{I}} q_{15}^{\mathrm{II}}\left(q_{15}^{\mathrm{I}}+q_{15}^{\mathrm{II}}\right)}{c_{44}^{\mathrm{II}} c_{44}^{\mathrm{I}}\left(\mu_{11}^{\mathrm{I}}+\mu_{11}^{\mathrm{II}}\right)+c_{44}^{\mathrm{I}}\left(q_{15}^{\mathrm{II}}\right)^{2}+c_{44}^{\mathrm{II}}\left(q_{15}^{\mathrm{I}}\right)^{2}} .
\end{gathered}
$$

In particular, for a fully permeable crack considered here, and two identical magneto or electroelastic planes polarized in opposite directions we have (from (94))

$$
K_{\mathrm{int}}^{D}=K_{\mathrm{int}}^{B}=0 .
$$

Note that the crack tip electric displacement $K_{\text {int }}^{D}$ and the electric displacement inside the crack $d_{0}$ exist only in the piezoelectric plane. Alternatively the crack tip magnetic induction intensity factor $K_{\text {int }}^{B}$ and the magnetic induction inside the crack $b_{0}$ exist only in the piezomagnetic plane. All quantities occur in the PEMO-elastic bimaterial.

\section{Results and Discussions}

In studying the fracture behaviour of the PEMO-elastic material, the field intensity factors are of significance. In this section, examples are given to illustrate the effects of material properties on the field intensity factor and the order of singularity.
TABLE 1: Relevant material properties $[14,15]$ and values of material parameters $m$ and $1 / m$.

\begin{tabular}{lccccc}
\hline & $\begin{array}{c}c_{44} \\
{\left[10^{9} \mathrm{~N} / \mathrm{m}^{2}\right]}\end{array}$ & $\begin{array}{c}e_{15} \\
{\left[\mathrm{C} / \mathrm{m}^{2}\right]}\end{array}$ & $\begin{array}{c}\varepsilon_{11} \\
{\left[10^{-9} \mathrm{C} / \mathrm{Vm}\right]}\end{array}$ & $m$ & $1 / m$ \\
\hline BaTiO $_{3}$ & 43,0 & 11,60 & 11,20 & 0,279 & 4,348 \\
PZT-5H & 35,3 & 17,00 & 15,10 & 0,542 & 1,844 \\
PZT-4 & 25,6 & 13,44 & 6,00 & 1,175 & 0,851 \\
P-7 & 25,0 & 13,50 & 17,10 & 0,430 & 2,325 \\
C-205 & 87,0 & 13,59 & 7,95 & 0,210 & 4,761 \\
PZT-PIC151 & 20,0 & 12,00 & 9,82 & 0,733 & 1,364 \\
\hline
\end{tabular}

5.1. Effect of Material Constants on the Singularity Order. We now consider the dependence of the singularity order on $2 \times 6$-constituent independent piezo-electromagnetoelastic constants. Although analytical evaluation of the relative sensitivities is possible, on the basis of the results presented above, it is rather cumbersome. Therefore, the sensitivity is evaluated here in other way.

Firstly, we assume that both materials are piezoelectric and $c_{44}^{\mathrm{II}}=\rho_{c} c_{44}^{\mathrm{I}}, e_{15}^{\mathrm{II}}=\rho_{e} e_{15}^{\mathrm{I}}$, and $\varepsilon_{11}^{\mathrm{II}}=\rho_{\varepsilon} \varepsilon_{11}^{\mathrm{I}}$, and analyze the situations
(a) $\rho_{c}$ changes and $\rho_{e}=\rho_{\varepsilon}=1$, that is, not change,
(b) $\rho_{e}$ changes and $\rho_{c}=\rho_{\varepsilon}=1$,
(c) $\rho_{\varepsilon}$ changes and $\rho_{c}=\rho_{e}=1$.

This states that it is analyzed that right half-plane is fixed, and left one contains a fictitious material with only changing $\rho_{c}$ or $\rho_{e}$ or $\rho_{\varepsilon}$.

(a) The changes of the ratio $\rho_{c}$ of $c_{44}^{\mathrm{II}}$ to $c_{44}^{\mathrm{I}}$ : we have

$$
\begin{aligned}
& \lambda=\frac{\left(1-\rho_{c}\right)(1+m)}{1+\rho_{c}+2 m}, \quad m=\left(\frac{\left(e_{15}\right)^{2}}{c_{44} \varepsilon_{11}}\right)^{\mathrm{I}}, \\
& |\lambda|<1, \quad \rho_{c}<3+\frac{2}{m} \quad \text { for } \rho_{e}=\rho_{\varepsilon}=1
\end{aligned}
$$

or

$$
\begin{aligned}
& \lambda=\frac{1-\rho_{c}}{1+\rho_{c}}-m, \\
& \lambda=0 \quad \text { for } \rho_{c}=\frac{1-m}{1+m}, \rho_{c}<\frac{2}{m}-1, \\
& 0<m<1 \quad \text { for } \rho_{e}=-1, \rho_{\varepsilon}=1 .
\end{aligned}
$$

Figure 3 shows the effects of varying elastic stiffness $\rho_{c}$ on $\lambda$ and $\alpha$ with unchanging piezoelectric and piezomagnetic constants $\rho_{e}=\rho_{\varepsilon}=1$ or $\rho_{e}=-1$ and $\rho_{\varepsilon}=1$. Note that $\lambda=0$ and $\alpha=1 / 2$ for $\rho_{e}=-1, \rho_{\varepsilon}=1$ and if $\left(c_{44}^{\mathrm{I}}-c_{44}^{\mathrm{II}}\right) c_{44}^{\mathrm{I}} \varepsilon_{11}^{\mathrm{I}}=$ $\left(c_{44}^{\mathrm{I}}+c_{44}^{\mathrm{II}}\right)\left(e_{15}^{\mathrm{I}}\right)^{2}, e_{15}^{\mathrm{II}}=-e_{15}^{\mathrm{I}}$ or if $\rho_{c}=1$ and $\rho_{e}=\rho_{\varepsilon}=1$. Note also that $\lambda\left(\rho_{e}=1, \rho_{\varepsilon}=1\right)>\lambda\left(\rho_{e}=-1, \rho_{\varepsilon}=1\right)$ for all of $\rho_{c}$.

The singularity order $\alpha$ is larger for two of the same ceramics poled in opposite directions together since $\alpha\left(\rho_{e}=\right.$ $\left.1, \rho_{\varepsilon}=1\right)<\alpha\left(\rho_{e}=-1, \rho_{\varepsilon}=1\right)$.

We take six kinds of particular piezoelectric ceramics as representatives, the relevant material constants and parameters $m$, and $1 / m$ of which are listed in Table 1 (with materials poling axes aligned in the positive $z$-direction). 


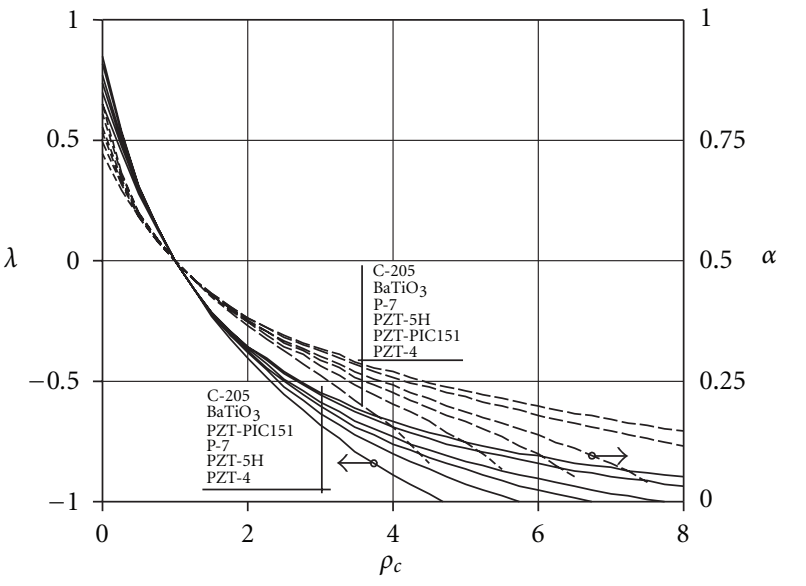

(a) Case I

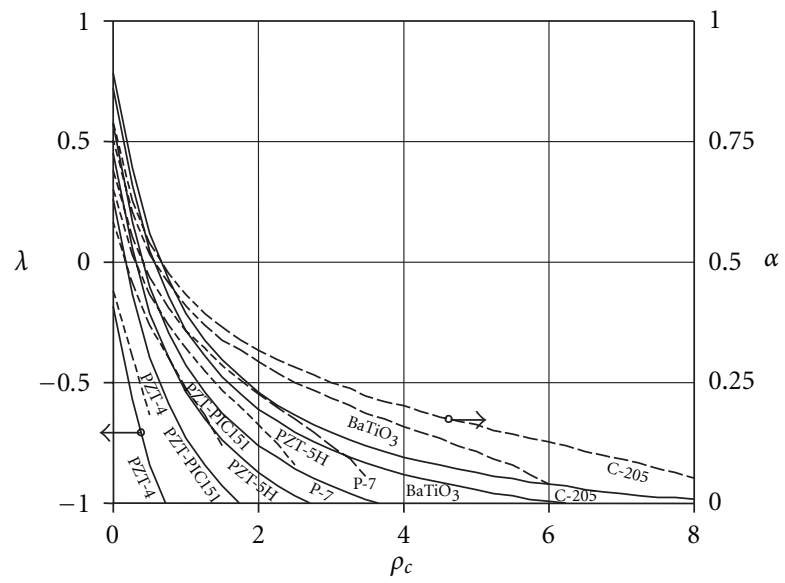

(b) Case II

Figure 3: Effect of $\rho_{c}$ on $\lambda$ and $\alpha$ with $\rho_{e}=\rho_{\varepsilon}=1$ (Case I) and $\rho_{e}=-1, \rho_{\varepsilon}=1$ (Case II).

(b) The changes of the ratio $\rho_{e}$ of $e_{15}^{\mathrm{II}}$ to $e_{15}^{\mathrm{I}}$ : we have

$$
\begin{aligned}
& \lambda=-\frac{\left(1-\rho_{e}\right)^{2}}{4 / m+\left(1+\rho_{e}\right)^{2}}, \quad \rho_{e}>-\frac{1}{m}=-\left(\frac{c_{44} \varepsilon_{11}}{\left(e_{15}\right)^{2}}\right)^{I}, \\
& \lambda_{\max }=0 \quad \text { for } \rho_{e}=1, \\
& \lambda_{\min }=-1-m \quad \text { for } \rho_{e}=-1-\frac{2}{m}<-\frac{1}{m}, \\
& \lambda=-1 \quad \text { for } \rho_{e}=-\frac{1}{m}, \\
& \lambda=-\frac{m}{4+m} \quad \text { for } \rho_{e}=0 \\
& \lambda=-m \quad \text { for } \rho_{e}=-1 .
\end{aligned}
$$

For $-1 / m<\rho_{e}<1$, the singularity parameter $\lambda$ increases from -1 to maximum $\lambda=0$ and for $\rho_{e}>1$ declines to -1 . Then the singularity parameter $\alpha$ varies between $(0,0,5)$, respectively. If both poling directions are opposite; that is, one is in the $z$-direction and second is in the $(-z)$ direction, then to satisfy the condition $\lambda>-1$ must hold $\left|e_{15}^{\mathrm{II}(-)}\right| / e_{15}^{\mathrm{I}(+)}<1 / m$ or $\left|e_{15}^{\mathrm{II}(-)}\right| e_{15}^{\mathrm{I}(+)}<c_{44}^{\mathrm{I}} \varepsilon_{11}^{\mathrm{I}}$. If the selection of $e_{15}^{\mathrm{II}}$ violates the condition $|\lambda|<1$, then the electroelastic field near the interface crack tip is dominant by either logarithmic singularity or is bonded. This situation seems unlikely, take place for realistic piezoelectric ceramics, and it is not beyond the scope of abilities of results of this paper.

(c) For $\rho_{\varepsilon}$ varying and other parameter unchanged, it is easily found that $\lambda=0$ and $\alpha=0,5$ for $\rho_{c}=\rho_{\varepsilon}=1$ and varying $\rho_{\varepsilon}$. But if $\rho_{e}=-1$, then

$$
\lambda=-\frac{2 m}{1+\rho_{\varepsilon}} ; \quad m=\left(\frac{\left(e_{15}\right)^{2}}{c_{44} \varepsilon_{11}}\right)^{\mathrm{I}} .
$$

Figure 5 shows the variation of $\lambda$ and $\alpha$ with the ratio $\rho_{\varepsilon}$ for $e_{15}^{\mathrm{II}(-)} / e_{15}^{\mathrm{I}(+)}=-1$.

The parameter $\lambda$ assumes negative values and increases from $-2 m$ to zero with $\rho_{\varepsilon}>0$. The singularity parameter $\alpha$ is positive and increases from $(1 / \pi) \arccos (2 m)$ to $1 / 2$ with $\rho_{\varepsilon}>0$. Note that $2 m$ must be less unity if $\rho_{\varepsilon}$ tends to zero or $m<1$ for $\rho_{\varepsilon}>1$. Some materials shown in Table 1 limit the range of $\rho_{\varepsilon}$; for example, PZT- 4 has $m=1,175$, and $\rho_{\varepsilon}$ must be larger 1,35 to ensure that $\lambda<-1$. Of course this situation is addressed to two piezoelectrics poled in opposite directions.

For piezomagnetic materials, the parameter $m$ is

$$
m=\left(\frac{\left(q_{15}\right)^{2}}{c_{44} \mu_{11}}\right)^{\mathrm{I}}
$$

and for magnetostrictive material $\mathrm{CoFe}_{2} \mathrm{O}_{4}$ assumes the value $m=0,0113$.

For $\mathrm{CoFe}_{2} \mathrm{O}_{4}$ we have

$$
\begin{gathered}
c_{44}=45,3 \mathrm{GPa}, \quad q_{15}=550 \mathrm{~N} / \mathrm{Am}, \\
\mu_{11}=590 \times 10^{-6} \mathrm{~N} / \mathrm{A}^{2} .
\end{gathered}
$$

The "relative sensitivity" analysis includes three cases:

(a) The changes of ratio $\rho_{c}$ of $c_{44}^{\mathrm{II}}$ to $c_{44}^{\mathrm{I}}$ : we have

$$
\lambda=\frac{1,0113\left(1-\rho_{c}\right)}{1,0226+\rho_{c}}, \quad \rho_{c}<20,7 \quad \rho_{q}=\rho_{\mu}=1
$$

or

$$
\lambda=\frac{1-\rho_{c}}{1+\rho_{c}}-0,0113, \quad \rho_{c}<16,7 \quad \rho_{q}=-1, \quad \rho_{\mu}=1 .
$$

Approximately,

$$
\lambda=\frac{1-\rho_{c}}{1+\rho_{c}}, \quad \rho_{c}<16 .
$$

For Magnetoelectroelastic composite $\mathrm{BaTiO}_{3}-\mathrm{CoFe}_{2} \mathrm{O}_{4}$ $\left(V_{f}=0,5\right)\left(q_{15}\right)^{2} / c_{44} \mu_{11}=0,005$ and $\left(e_{15}\right)^{2} / c_{44} \varepsilon_{11} \approx 0,135$.

Figure 6 shows the effect of $\rho_{c}$ on $\lambda$ and $\alpha$ for $\mathrm{CoFe}_{2} \mathrm{O}_{4}$ magnetostrictive ceramic. 


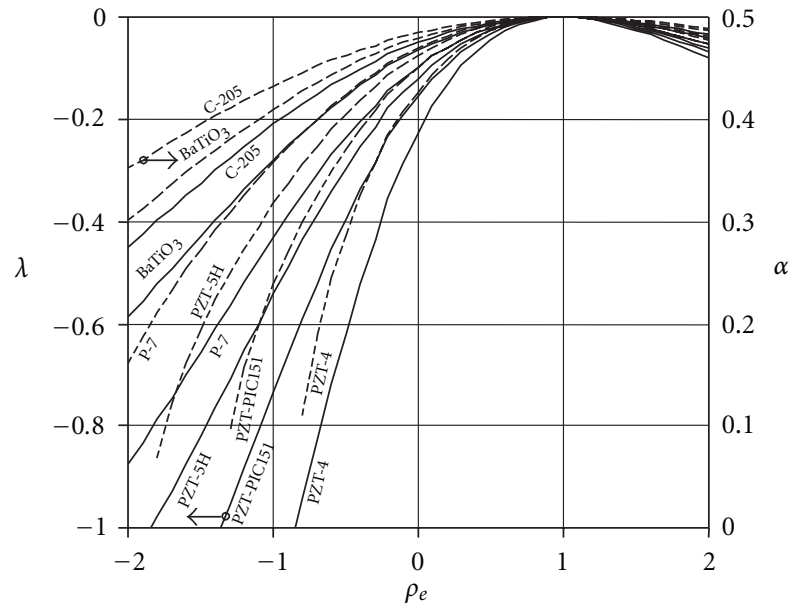

Figure 4: Effects of $\rho_{e}$ on $\lambda$ and $\alpha$ with $\rho_{c}=\rho_{\varepsilon}=1\left(\rho_{e}>0\right.$ or $\rho_{e}<0$ denote piezoelectric ceramics poled parallel to or antiparallel to the $z$-axis, respectively, i.e., $\rho_{e}=-1$ denotes that $\left.e_{15}^{\mathrm{I}}=-e_{15}^{\mathrm{II}}=e_{15}\right)$.

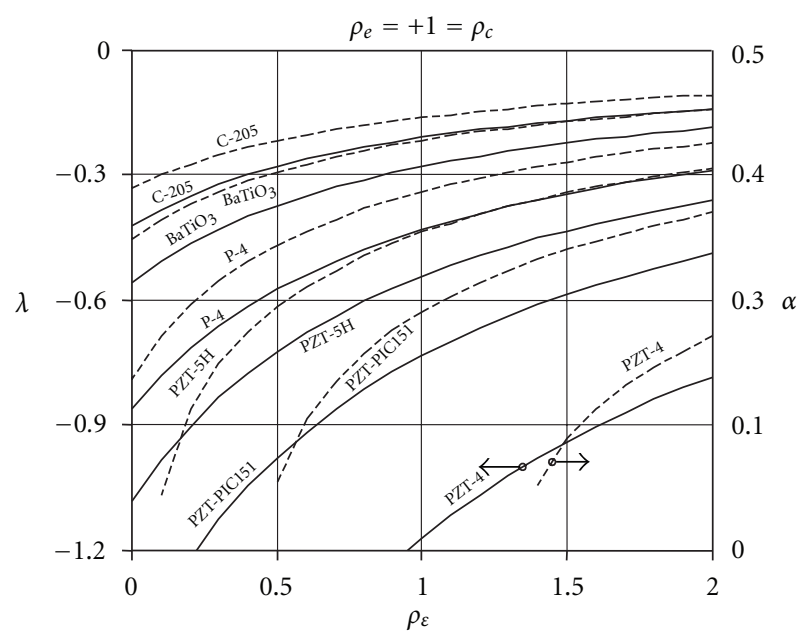

FIGURE 5: Effect of $\rho_{\varepsilon}$ on $\lambda$ and $\alpha$ with $\rho_{c}=1$ when $\rho_{e}=e_{15}^{\mathrm{II}(-)} / e_{15}^{\mathrm{I}(+)}=$ -1 ; for $\rho_{e}=1$, we have $\lambda=0$ and $\alpha=0,5$.

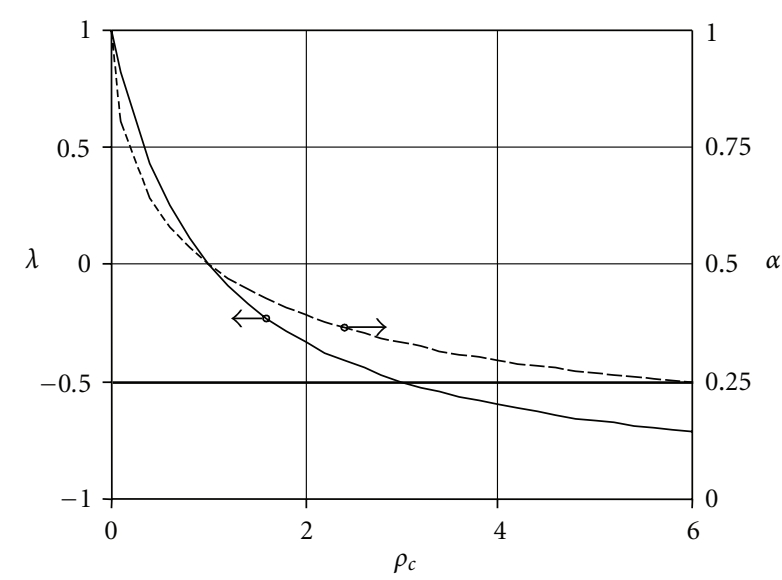

Figure 6: Effect of $\rho_{c}$ on $\lambda$ and $\alpha$ for $\mathrm{CoFe}_{2} \mathrm{O}_{4}, \rho_{\mu}=1$ and $\rho_{q}=1$ or $\rho_{q}=-1$.

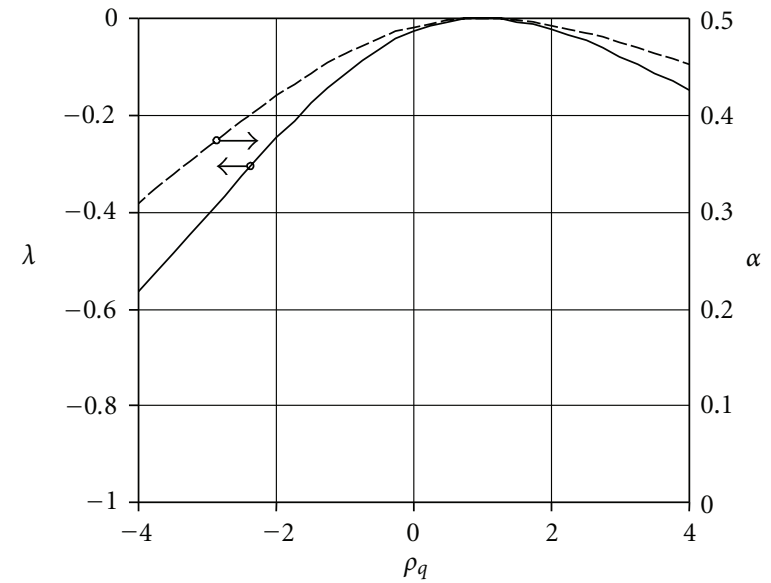

Figure 7: Effects of $\rho_{q}$ on $\lambda$ and $\alpha$ for $\mathrm{CoFe}_{2} \mathrm{O}_{4}, \rho_{c}=\rho_{\mu}=1$.

For both poling directions, the values of $\lambda$ and $\alpha$ are the same.

(b) The changes of the ratio $\rho_{q}$ of $q_{15}^{\mathrm{II}}$ to $q_{15}^{\mathrm{I}}$ : we have

$$
\begin{aligned}
& \lambda=-\frac{\left(1-\rho_{q}\right)^{2}}{35,4+\left(1+\rho_{q}\right)^{2}}, \quad \rho_{q}>-8,85, \\
& \lambda_{\max }=0 \quad \text { for } \rho_{q}=1, \\
& \lambda=-1 \quad \text { for } \rho_{q}=-8,85 .
\end{aligned}
$$

Figure 7 shows the effect of $\rho_{q}$ on $\lambda$ and $\alpha$ for $\mathrm{CoFe}_{2} \mathrm{O}_{4}$ ceramic.

(c) The changes of the ratio $\rho_{\mu}$ of $\mu_{15}^{\mathrm{II}}$ to $\mu_{15}^{\mathrm{I}}$ : we have

$$
\begin{aligned}
& \lambda=-\frac{0,0226}{1+\rho_{\mu}} \text { for } \rho_{q}=-1, \\
& \lambda=0 \quad \text { for } \rho_{q}=1, \quad \text { always } \rho_{c}=1 .
\end{aligned}
$$

Figure 8 shows the effect of $\rho_{\mu}$ on $\lambda$ and $\alpha$ for $\mathrm{CoFe}_{2} \mathrm{O}_{4}$.

5.2. Effect of Material Constants on the Field Intensity Factors. The material constants also affect the intensity factors. Figure 9 presents the variation of normalized SIFs $k_{\text {hom }}^{\tau}$ and $k_{\text {int }}^{\tau}$ defined by (89) and (90) which depend on $\alpha$ and $\lambda$

$$
\begin{gathered}
k_{\text {hom }}^{\tau}=\frac{2 \alpha}{\sqrt{1+\lambda}}, \\
k_{\text {int }}^{\tau}=2^{3 \alpha-1 / 2} \frac{1-\alpha}{1+\lambda} \sqrt{1-\lambda} .
\end{gathered}
$$

For $0<\alpha<1 k_{\text {hom }}^{\tau}$ increasing monotonously from $2 \sqrt{2} / \pi$ through 1 to $\sqrt{2}$ as $\alpha$ tends to zero and equals $1 / 2$ and 1 , respectively. From Figures 8 and 2, one can observe that the effect of $\rho_{c}$ on $k_{\text {int }}^{\tau}$ is more evident than that on $k_{\mathrm{hom}}^{\tau}$. Moreover, $\rho_{c}$ increased the singularity parameter $\alpha$ that decreases (see Figure 3), and $k_{\text {int }}^{\tau}$ rises suddenly, while $k_{\text {hom }}^{\tau}$ falls down slightly. For $\rho_{c}<1$ and $\rho_{e}=1$ or $\rho_{c}<(1-$ $m) /(1+m)$ and $\rho_{e}=-1$, we have $\alpha>1 / 2$. This means 


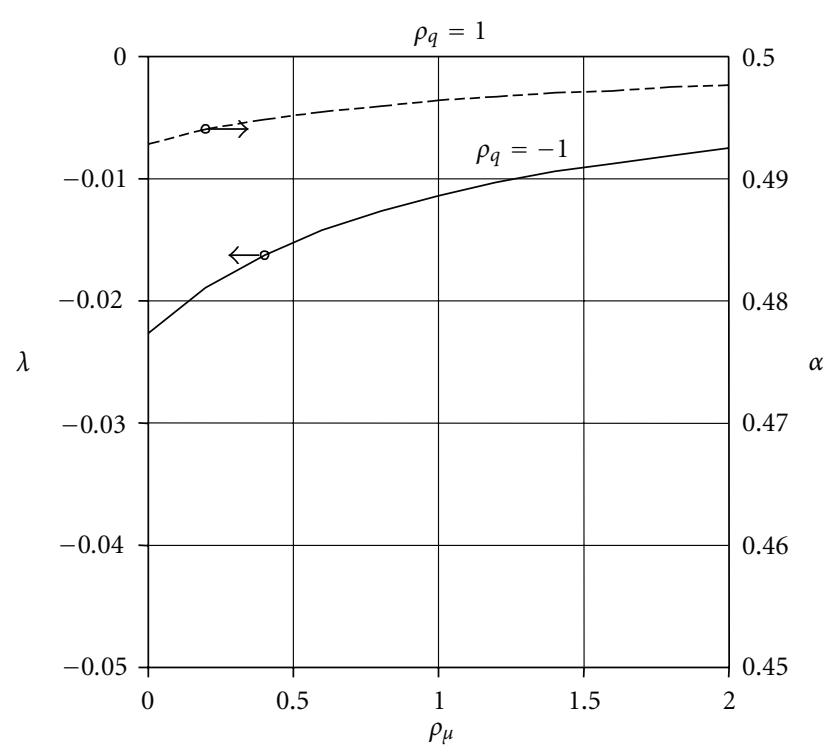

Figure 8: Effects of $\rho_{\mu}$ on $\lambda$ and $\alpha$ for $\mathrm{CoFe}_{2} \mathrm{O}_{4}, \rho_{q}=1$ or $\rho_{q}=-1$ and $\rho_{c}=1$.

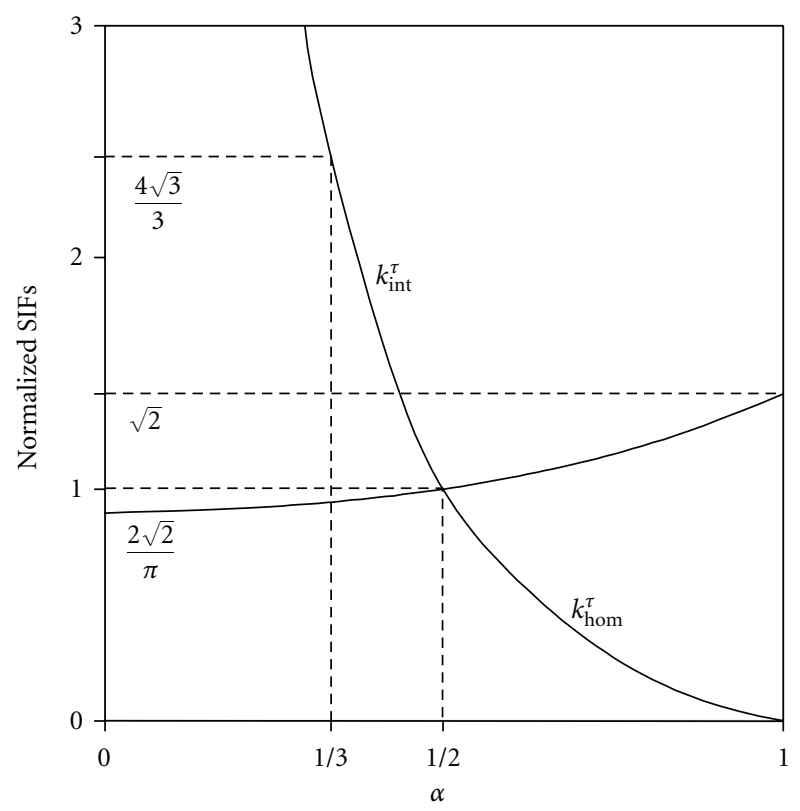

Figure 9: Normalized SIFs as a function of $\alpha$.

that if piezoelectric II is more elastically complaisant than piezoelectric ceramic I, in this case $k_{\mathrm{hom}}^{\tau}>k_{\mathrm{int}}^{\tau}$. On the other hand, for $\rho_{c}>1$, which gives $\alpha<1 / 2$, meaning that piezoelectric II is stiffer than piezoelectric I in this case $k_{\text {hom }}^{\tau}<k_{\text {int }}^{\tau}$. From the Figures 3, 4, and 5, we see that the range $0<\alpha<1 / 2$ corresponds to $\rho_{c}>1$ or $\rho_{c}>(1-m) /(1+m)$ (in the case $\rho_{e}=-1$ ), $\rho_{e}>-1 / m$, and $\rho_{\varepsilon}>0$. Then always $k_{\text {int }}^{\tau}>k_{\text {hom }}^{\tau}$. The range $1 / 2<\alpha<1$ is for $0<\rho_{c}<1$ or $0<\rho_{c}<(1-m) /(1+m)$ (in the case $\left.\rho_{e}=-1\right)$. Then, $k_{\text {int }}^{\tau}<k_{\text {hom }}^{\tau}$ for all of $\alpha$.
Note that the case $\alpha=1, \lambda=1$ gives the limiting values $k_{\text {int }}^{\tau}=0$ and $k_{\text {hom }}^{\tau}=\sqrt{2}$ which gives

$$
K_{\text {int }}^{\tau}=0, \quad K_{\text {hom }}^{\tau}=\tau_{0}^{\mathrm{I}} \sqrt{\pi a} .
$$

This is the solution for edge crack of length $a$.

The normalized intensity factors for strain, electric displacement, magnetic induction, electric field, and magnetic field at the interface crack tip are defined by (78) and by the following formula:

$$
k_{\mathrm{int}}^{q}=\frac{c_{44}^{\mathrm{I}}}{\tau_{o}^{\mathrm{I}}} \frac{K_{\mathrm{int}}^{q}}{(\pi a / 2)^{\alpha}},
$$

where $q$ stands for one of $\gamma, D, B, E$, and $H$.

Then we have

$$
\begin{aligned}
& {\left[k_{\text {int }}^{\gamma} ; k_{\text {int }}^{D} ; k_{\text {int }}^{B} ; k_{\text {int }}^{E} ; k_{\text {int }}^{H}\right]} \\
& \quad=\frac{\sqrt{2}(1-\alpha) 8^{\alpha}}{(1+\lambda) \sqrt{1-\lambda}}\left[\lambda_{\gamma} ; e_{15}^{\mathrm{I}} \lambda_{D} ; q_{15}^{\mathrm{I}} \lambda_{B} ; \lambda_{E} ; \lambda_{H}\right],
\end{aligned}
$$

respectively.

Of course, the normalized intensity factors satisfy the constitutive equations (5), that is,

$$
\left[k_{\text {int }}^{\tau} ; k_{\text {int }}^{D} ; k_{\text {int }}^{B}\right]=C^{\mathrm{II}}\left[k_{\text {int }}^{\gamma} ;-k_{\text {int }}^{E} ;-k_{\text {int }}^{H}\right]
$$

with the matrix (6) or inverse form with the use of matrix $\left(C^{\mathrm{II}}\right)^{-1}$, defined by $(17)$.

The analysis above implies that, for the magnetically (or electrically) permeable interfacial cracks, the applied magnetic (or electric) loadings have no influence on the fracture behaviours of the crack tips.

Figures 10 and 11 are devoted to the variation of $k_{\text {int }}^{\tau}$ and $k_{\text {hom }}^{\tau}$.

We have

$$
\begin{aligned}
& k_{\text {hom }}^{\tau}=\frac{2 \sqrt{2}}{\pi} \quad \text { for } \rho_{c}=3+\frac{2}{m} \quad\left(\rho_{e}=\rho_{\varepsilon}=1\right) \\
& \text { or } \rho_{c}=\frac{2}{m}-1 \quad\left(\rho_{e}=-1, \rho_{\varepsilon}=1\right) .
\end{aligned}
$$

The figures show that the normalized stress intensity factor in homogeneous solid is only weakly dependent on the elastic constants and dielectric permeabilities. In contrast $k_{\text {int }}^{\tau}$ strongly depends on $\rho_{c}$ and $\rho_{e}$. This is consistent with physical considerations; for large difference of piezocoefficients $\rho_{e}<0$ or $\rho_{e}>1$ the $k_{\text {int }}^{\tau}$ are larger than $k_{\text {hom }}^{\tau}$ (Figure 11). From the Figure 10 it can be shown that the piezoelectric ceramic II is more complaisant than piezoelectric ceramic I $\left(\rho_{c}<1\right)$, then $k_{\text {hom }}^{\tau}>k_{\text {int }}^{\tau}$. In contrast if $\rho_{c}>1$ meaning that piezoelectric ceramic II is stiffer than piezoelectric ceramic I, in this case $k_{\mathrm{hom}}^{\tau}<k_{\mathrm{int}}^{\tau}$.

Other normalized field intensity factors are presented on Figures 12 and 13.

The $k_{\text {int }}^{E}$ is equal to zero for $\rho_{c}=1$ (Figure 12) and for $\rho_{e}=1$ (Figure 13). From (81) one finds that $k_{\text {int }}^{E}=0$ occurs only when $c_{44}^{\mathrm{II}} / c_{44}^{\mathrm{I}}=e_{15}^{\mathrm{II}} / e_{15}^{\mathrm{I}}$. In Figure 13 we see that $\rho_{e}$ has a strong influence on $k_{\text {int }}^{D}$ and $k_{\text {int }}^{E}$ and $k_{\text {int }}^{\gamma}$ if $\rho_{e}<1$, and when $\rho_{e}=1, k_{\text {int }}^{\gamma}=1, k_{\text {int }}^{E}=0$, and $k_{\text {int }}^{D}=e_{15}^{I}$, as expected. 


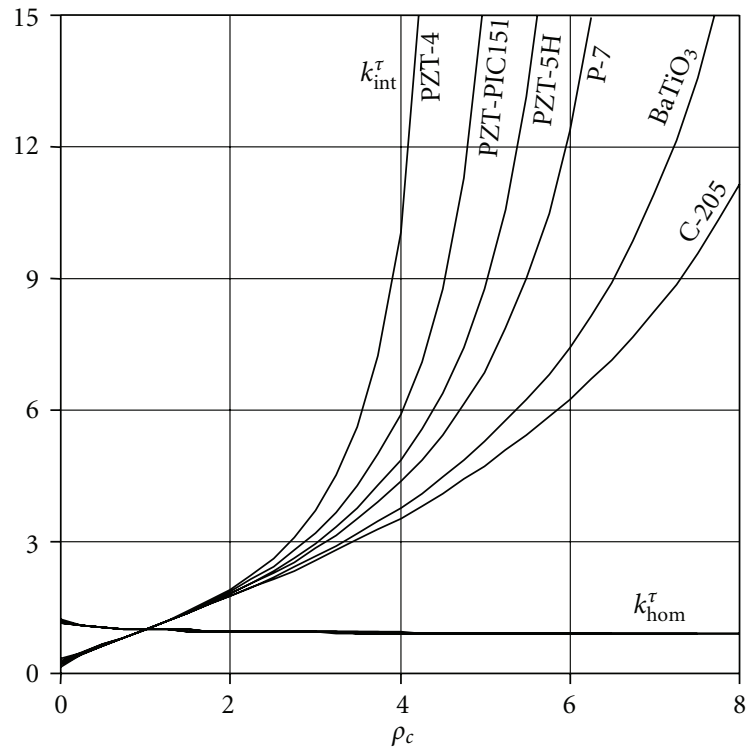

(a) Case I

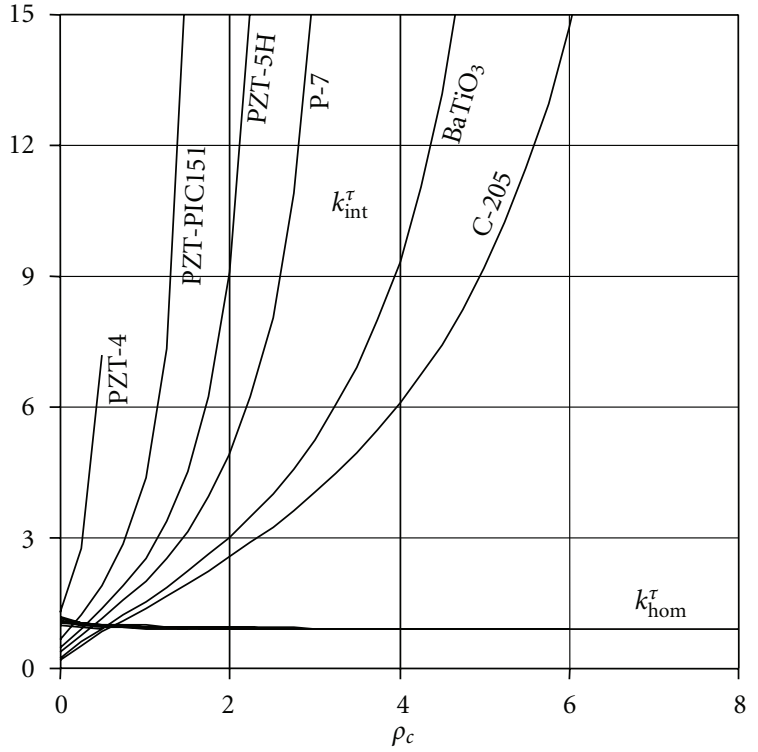

(b) Case II

FIGURE 10: Normalized SIFs as a function of $\rho_{c}$ with $\rho_{e}=\rho_{\varepsilon}=1$ (Case I) and $\rho_{e}=-1, \rho_{\varepsilon}=1$ (Case II).

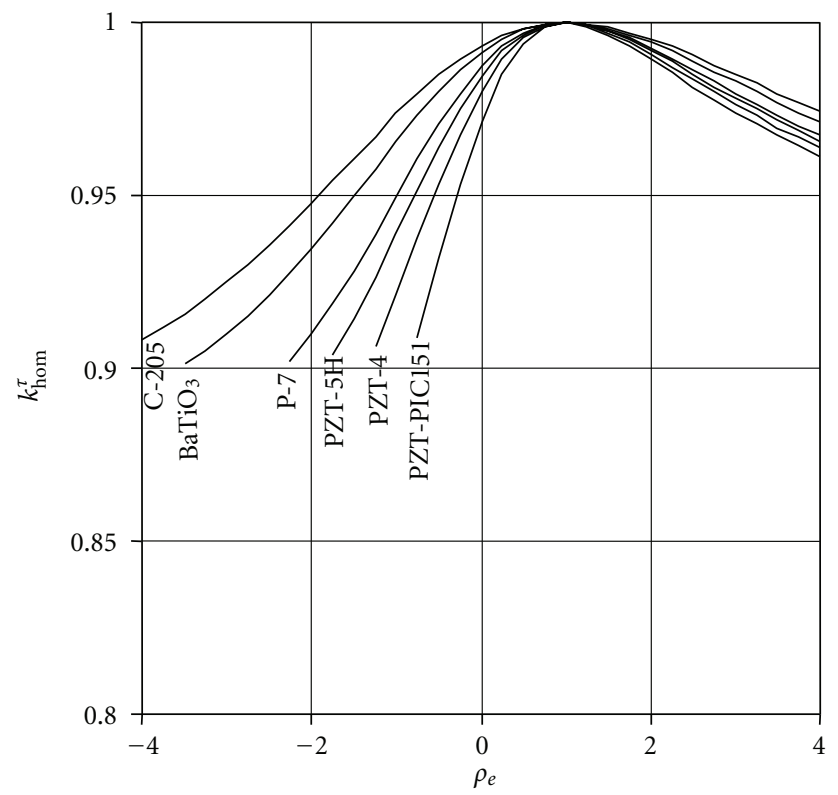

(a)

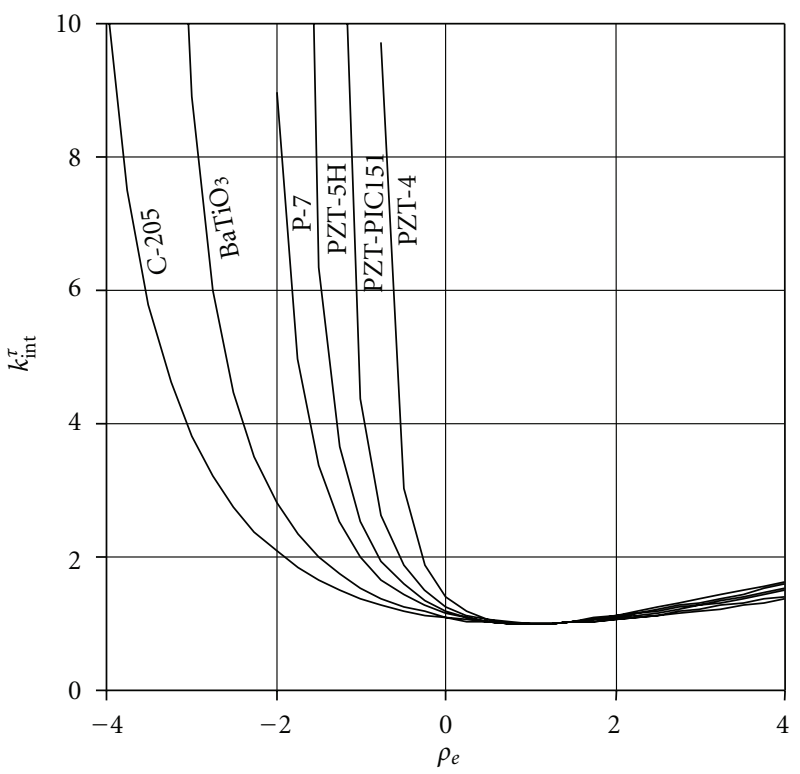

(b)

FIGURE 11: Normalized SIFs as a function of $\rho_{e}$ with $\rho_{c}=\rho_{\varepsilon}=1$.

Figure 14 presents the variation of normalized ERRs, $G / G_{\text {hom }}$ obtained from (87) with the use of (107).

There are two states where $G=G_{\text {hom }}$. The first state, in which $\alpha=1 / 2$ and $\lambda=0$, that is, $c_{44}^{\mathrm{II}}=c_{44}^{\mathrm{I}}$, corresponds to crack in monolithic medium (no bi-material). The second state, in which $\alpha$ and $\lambda$ tend to unity, corresponds to edge crack problem (the second material is air). For $\alpha>1 / 2$ ERRs decrease weakly from 1 to 0,69 for $\alpha=3 / 4$ and later increase to unity for $\alpha \rightarrow 1$. In this case the piezoelectric ceramic II is more elastically complaisant. The range $0<\alpha<1 / 2$ corresponds to the following cases: $\rho_{c}>1$ or $\rho_{c}>(1-m) /(1+$ $m$ ) (in the case $\rho_{e}=-1$ ); $\rho_{e}>-1 / m$ and $\rho_{\varepsilon}>0$ (for any $\varepsilon$ ). Then always $G>G_{\text {hom }}$ and piezoelectric II are stiffer than piezoelectric I. Similar conclusions may be formulated for magnetostrictive material, changing material parameters $e_{15}$ and $\varepsilon_{11}$ by $q_{15}$ and $\mu_{11}$, respectively.

5.2.1. A Crack between a Piezoelectric Material and a Piezomagnetic Material. Magnetoelectroelastic materials usually 


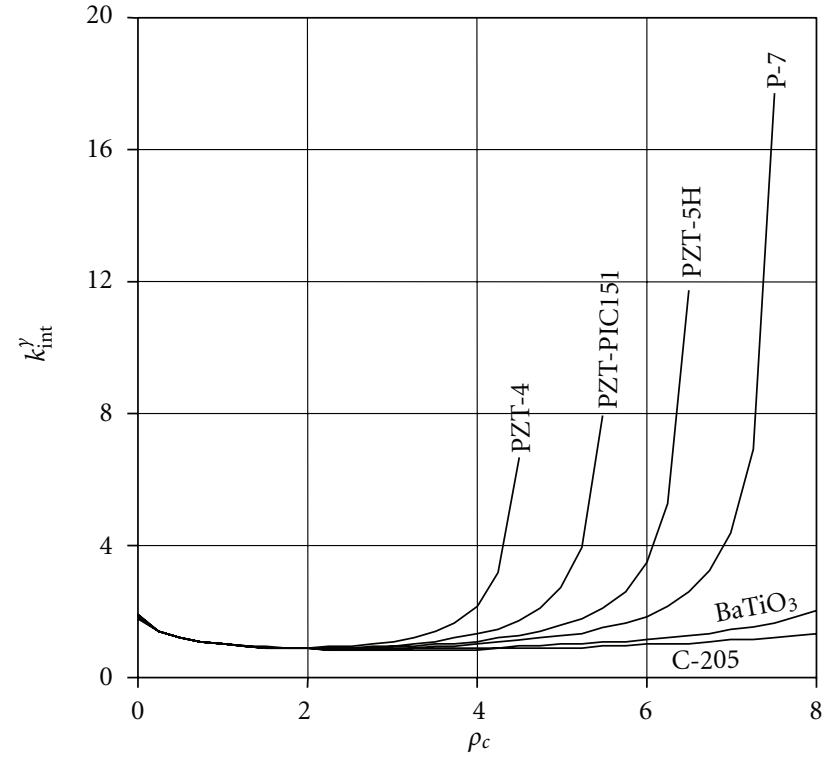

(a)

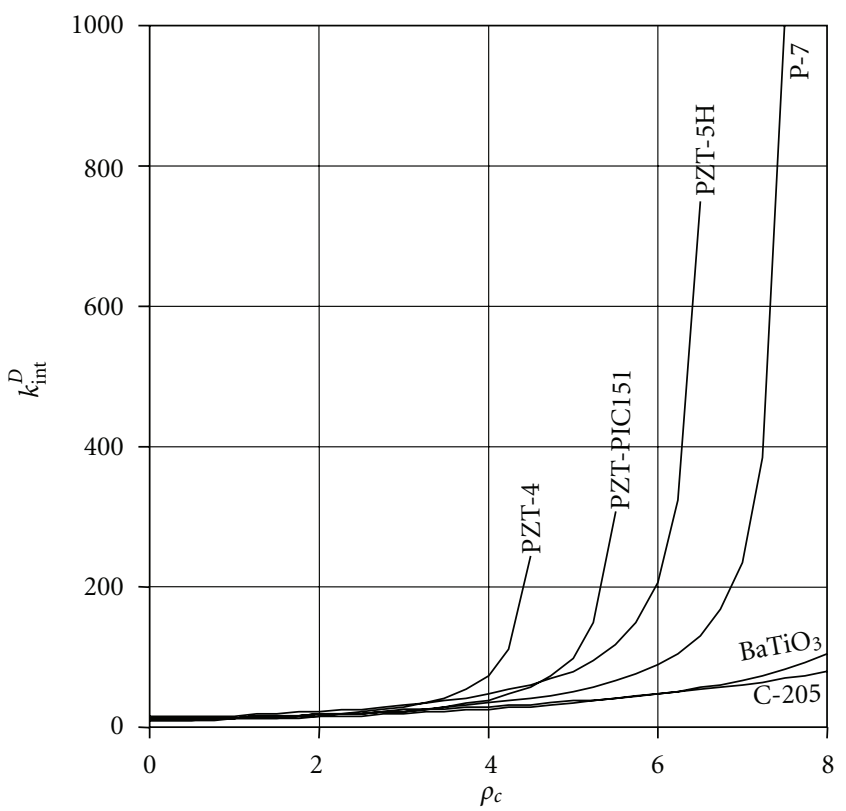

(b)

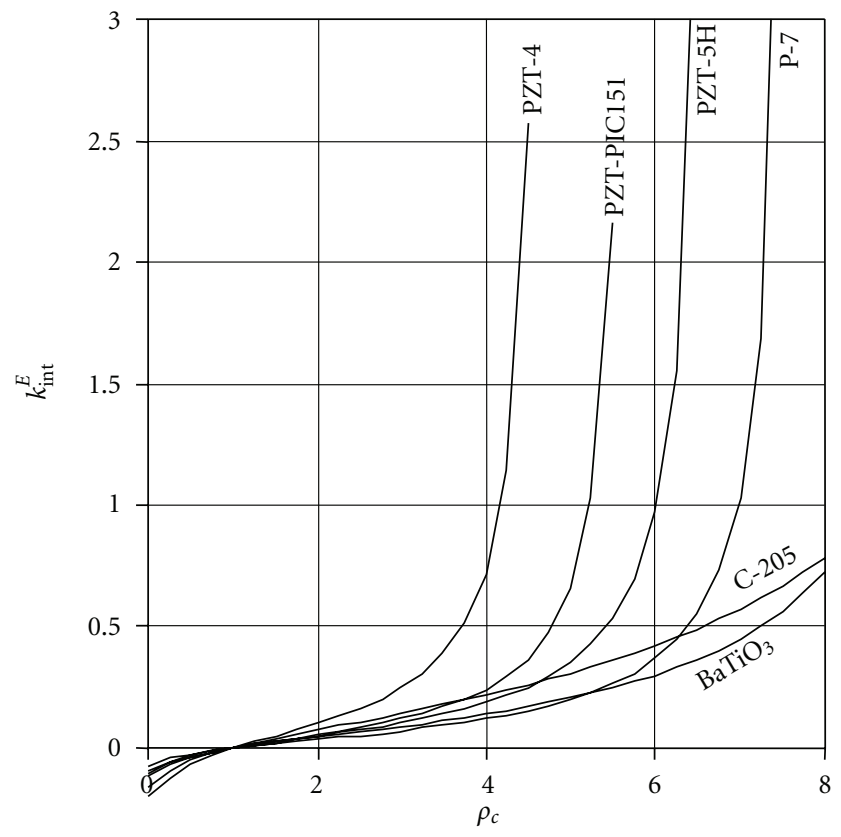

(c)

FIgURE 12: Variation of $k_{\text {int }}^{\gamma}, k_{\text {int }}^{D}\left(\right.$ in $\mathrm{C} / \mathrm{m}^{2}$ ) and $k_{\text {int }}^{E}$ (in $10^{6} \mathrm{kV} / \mathrm{m}$ ) against $\rho_{c}$ with $\rho_{e}=\rho_{\varepsilon}=1$.

comprise alternating piezoelectric medium and piezomagnetic medium. Here, we consider a special case. This is a right medium I that is a piezoelectric and the left medium II is a piezomagnetic (Case I) or inversely (Case II). The material constants of the piezoelectric medium (No. I) and piezomagnetic medium (No. II) have the following values [16-18]:

$\mathrm{BaTiO}_{3}$-piezoelectric (barium titanate)

$$
c_{44}^{\mathrm{I}}=43 \times 10^{9} \mathrm{Nm}^{-2}, \quad e_{15}^{\mathrm{I}}=11,6 \mathrm{Cm}^{-2},
$$

$$
\begin{array}{r}
q_{15}^{\mathrm{I}}=0, \quad \varepsilon_{11}^{\mathrm{I}}=11,2 \times 10^{-9} \mathrm{CV}^{-1} \mathrm{~m}^{-1}, \\
d_{11}^{\mathrm{I}}=0, \quad \mu_{11}^{\mathrm{I}}=5,0 \times 10^{-6} \mathrm{NA}^{-2}
\end{array}
$$

$\mathrm{CoFe}_{2} \mathrm{O}_{4}$-piezomagnetic (cobalt iron oxide)

$$
c_{44}^{\mathrm{II}}=45,3 \times 10^{9} \mathrm{Nm}^{-2}, \quad e_{15}^{\mathrm{II}}=0,
$$

$q_{15}^{\mathrm{II}}=550 \mathrm{NA}^{-1} \mathrm{~m}^{-1}, \quad \varepsilon_{11}^{\mathrm{II}}=0,08 \times 10^{-9} \mathrm{CV}^{-1} \mathrm{~m}^{-1}$,

$d_{11}^{\mathrm{II}}=0, \quad \mu_{11}^{\mathrm{II}}=590 \times 10^{-6} \mathrm{NA}^{-2}$. 


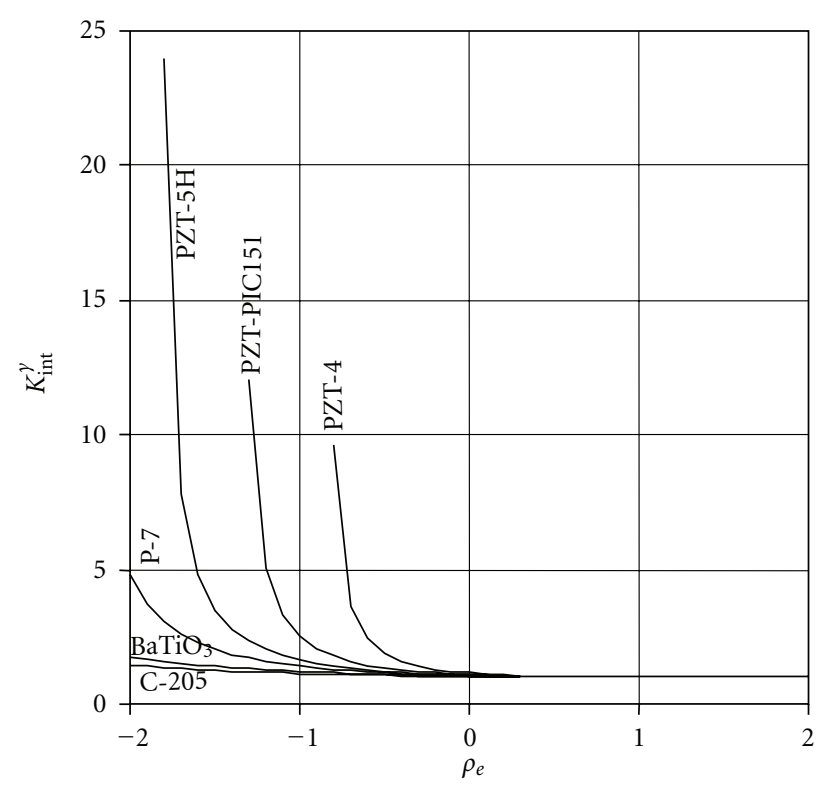

(a)

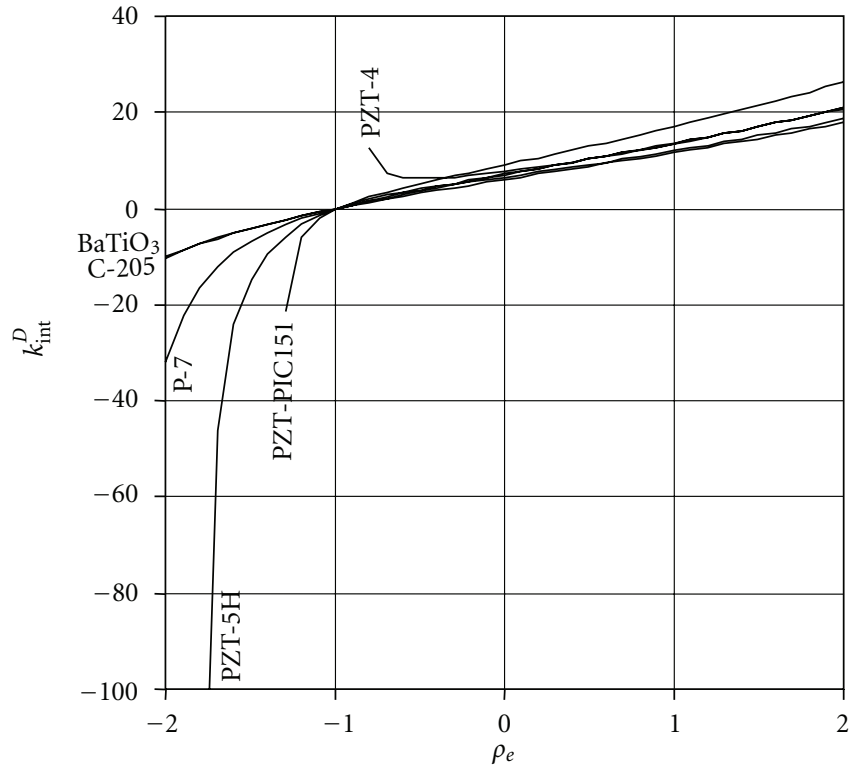

(b)

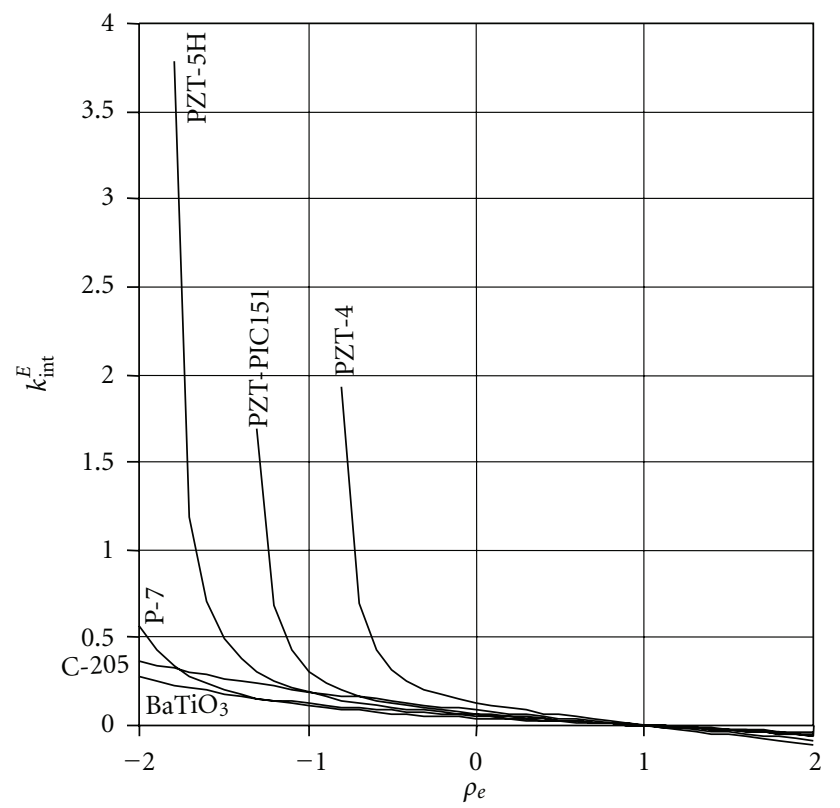

(c)

FIGURE 13: Variation of $k_{\text {int }}^{\gamma}, k_{\text {int }}^{D}\left(\right.$ in $\left.\mathrm{C} / \mathrm{m}^{2}\right)$ and $k_{\text {int }}^{E}$ (in $10^{6} \mathrm{kV} / \mathrm{m}$ ) against $\rho_{e}$ with $\rho_{c}=\rho_{\varepsilon}=1$.

The material parameter (57) assumes the values

$$
\lambda=1-\frac{2}{c_{44}^{J} /\left(c_{44}^{\mathrm{I}}+\left(e_{15}^{\mathrm{I}}\right)^{2} /\left(\varepsilon_{11}^{\mathrm{I}}+\varepsilon_{11}^{\mathrm{II}}\right)\right)+c_{44}^{J} /\left(c_{44}^{\mathrm{II}}+\left(q_{15}^{\mathrm{II}}\right)^{2} /\left(\mu_{11}^{\mathrm{I}}+\mu_{11}^{\mathrm{II}}\right)\right)} ; \quad J=\mathrm{I}, \mathrm{II} .
$$

where $c_{44}^{J}$ is the shear modulus of the cracked material, for Case I and Case II, respectively. We have

$$
\lambda=\left\{\begin{array}{ll}
-0,1618, & \text { Case I } \\
-0,1028, & \text { Case II }
\end{array} \quad \alpha= \begin{cases}0,4483, & \text { Case I } \\
0,4672, & \text { Case II. }\end{cases}\right.
$$




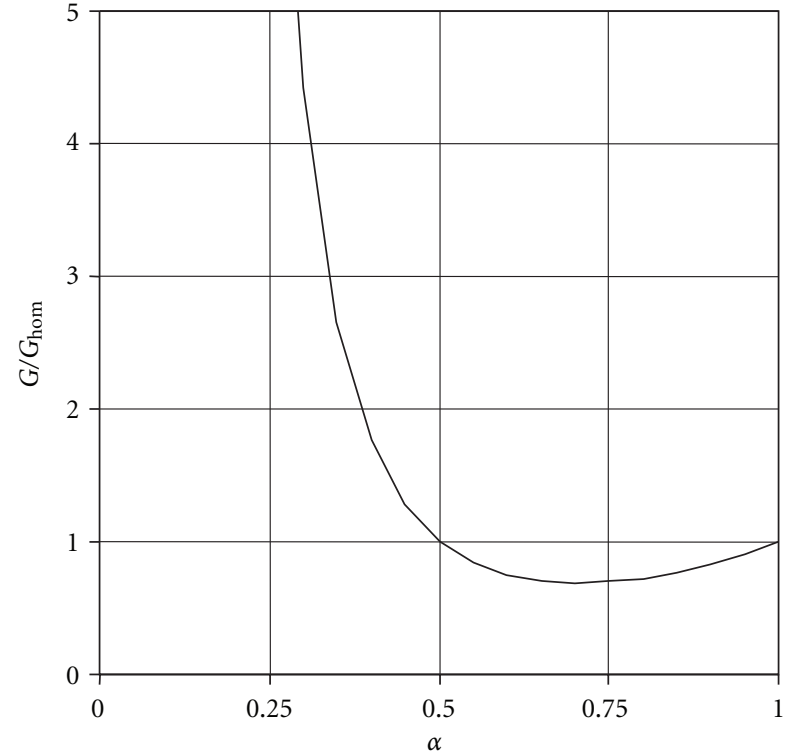

FIgURE 14: Normalized ERRs, $G / G_{\text {hom }}$ as a function of $\alpha: G / G_{\text {hom }}=$ $\left(2 \alpha^{2} /(1+\lambda)\right)\left[1+8^{2 \alpha-1}(1-1 / \alpha)^{2}(1-\lambda) /(1+\lambda)\right], \lambda=-\cos (\pi \alpha)$, $\alpha=3 / 4, \lambda=1 / \sqrt{2}, G / G_{\text {hom }}=(11 \sqrt{2}-10) / 8, \alpha=1 / 4, \lambda=-1 / \sqrt{2}$, $G / G_{\text {hom }}=(67+47 \sqrt{2}) / 16$.

The energy release rates are obtained as follows

$$
G= \begin{cases}\pi a(15,0) \times 10^{-12}\left(\tau_{0}^{\mathrm{I}}\right)^{2} \times\left[\mathrm{m}^{2} / \mathrm{N}\right], & \text { Case I } \\ \pi a(12,9) \times 10^{-12}\left(\tau_{0}^{\mathrm{I}}\right)^{2} \times\left[\mathrm{m}^{2} / \mathrm{N}\right], & \text { Case II. }\end{cases}
$$

For "homogenous" composite $\mathrm{BaTiO}_{3} / \mathrm{CoFe}_{2} \mathrm{O}_{4}$ with the ratio roughly $50: 50$, we have with the use of arithmetic mean $c_{44}=44,15 \times 10^{9} \mathrm{Nm}^{-2}$, and $G_{\text {hom }}$ assumes the value

$$
G_{\mathrm{hom}}=\pi a\left(11,4 \times 10^{-12}\left(\tau_{0}^{\mathrm{I}}\right)^{2}\right) \times\left[\mathrm{m}^{2} / \mathrm{N}\right] .
$$

We see that ERRs for bi-materials cannot be determined by the mixture rule since it is a significant new feature in interface crack problem considered in this paper.

Obviously for piezoelectric/piezomagnetic composite (I/II) is $\mu_{11}^{\mathrm{I}} \ll \mu_{11}^{\mathrm{II}}$ and $\varepsilon_{11}^{\mathrm{II}} \ll \varepsilon_{11}^{\mathrm{I}}$, and (115) reduces to the following formula:

$$
\lambda=1-\frac{\tilde{c}_{44}^{*}}{c_{44}^{J}} ; \quad J=\mathrm{I}, \mathrm{II},
$$

where $\widetilde{c}_{44}^{*}$ is the harmonic mean of the piezoelectric and piezomagnetic stiffened elastic constants $\tilde{c}_{44}^{I}$ and $\tilde{c}_{44}^{\mathrm{II}}$ defined as follows:

$$
\frac{1}{\widetilde{c}_{44}^{*}}=\frac{1}{2}\left(\frac{1}{\widetilde{c}_{44}^{I}}+\frac{1}{\widetilde{c}_{44}^{\mathrm{II}}}\right)
$$

where

$$
\tilde{c}_{44}^{\mathrm{I}}=c_{44}^{\mathrm{I}}+\frac{\left(e_{15}^{\mathrm{I}}\right)^{2}}{\varepsilon_{11}^{\mathrm{I}}}, \quad \tilde{c}_{44}^{\mathrm{II}}=c_{44}^{\mathrm{II}}+\frac{\left(q_{15}^{\mathrm{II}}\right)^{2}}{\mu_{11}^{\mathrm{II}}} .
$$

Using (119) to (121), we obtain that

$$
\begin{gathered}
\lambda=\left\{\begin{array}{ll}
-0,1626, & \text { Case I } \\
-0,1036, & \text { Case II }
\end{array} \quad \alpha= \begin{cases}0,4480, & \text { Case I, } \\
0,4670, & \text { Case II, }\end{cases} \right. \\
G= \begin{cases}\pi a(15,0) \times 10^{-12}\left(\tau_{0}^{\mathrm{I}}\right)^{2} \times\left[\mathrm{m}^{2} / \mathrm{N}\right], & \text { Case I, } \\
\pi a(12,9) \times 10^{-12}\left(\tau_{0}^{\mathrm{I}}\right)^{2} \times\left[\mathrm{m}^{2} / \mathrm{N}\right], & \text { Case II. }\end{cases}
\end{gathered}
$$

\section{Conclusions}

A crack perpendicular to and terminating at the interface of two bonded dissimilar piezo-electromagnetoelastic media are studied in this paper. Analytical solutions and numerical simulations suggest the following conclusions.

(i) Closed form solution has been obtained for a crack between two dissimilar magneto electro-elastic ceramics. The crack is localized in one materials, and its one tip lies on the interface. Expressions for the crack-tip field intensity factors, the electromagnetic fields inside the crack, are given for electrically and magnetically permeable crack assumptions.

(ii) The energy release rate can be explicitly expressed in terms of the intensity factors. It is affected by electricmagnetic properties of the constituents of the bimaterial media. The normalized energy release rate is unity for homogeneous medium $\left(\rho_{c}=1\right)$ and for edge crack $\left(\rho_{c}=0\right)$ and assumes minimum value 0,69 for $\rho_{c}=3-2 \sqrt{2}=0,18$. If $\rho_{c}$ tends to infinity, also this quantity tends to infinity (the interface is clamped).

(iii) For two identical Magnetoelectroelastic planes polarized in opposite directions, we have $K_{\text {int }}^{D}=0=K_{\text {int }}^{B}$.

(iv) At interface we have $K_{\text {int }}^{E}=0$ when $c_{44}^{\mathrm{II}} / c_{44}^{\mathrm{I}}=e_{15}^{\mathrm{II}} / e_{15}^{\mathrm{I}}$, while $K_{\text {int }}^{H}=0$ if $c_{44}^{\mathrm{II}} / c_{44}^{\mathrm{I}}=q_{15}^{\mathrm{II}} / q_{15}^{\mathrm{I}}$.

(v) Application of electric and magnetic fields do not alter the stress intensity factors; they depend on the elastic, electric, and magnetic constants of bimaterial ceramic.

(vi) The coupling between electromagnetic fields and mechanical field leads to existing electric displacement and magnetic induction intensity factors at the crack tip, which respond to the applied stress intensity factor.

(vii) If magnetic effects are neglected, the result of the stress intensity factors is the same as the solution for the piezoelectric materials given by Li and Wang [9], but $k_{\text {int }}^{E}$ differs in sign.

The results could be of particular interest to the analysis and design of smart sensors and actuators constructed from Magnetoelectroelastic composite laminates. Nowadays, electromagnetoelastic coupled multiphase composite has wide range applications in science and engineering such as space planes, supersonic air planes, rockets, missiles nuclear fusion, reactors, and submarines. 


\section{References}

[1] R. Li and G. A. Kardomateas, "The mode III interface crack in piezo-electro-magneto-elastic dissimilar bimaterials," ASME Journal of Applied Mechanics, vol. 73, no. 2, pp. 220-227, 2006.

[2] R. Li and G. A. Kardomateas, "The mixed modes I and II interface crack in piezo-electro-elastic anisotropic bi-materials," ASME Journal of Applied Mechanics, vol. 74, pp. 614627, 2007.

[3] B. L. Wang and Y. W. Mai, "Closed-form solution for an antiplane interface crack between two dissimilar magnetoelectroelastic layers," ASME Journal of Applied Mechanics, vol. 73, no. 2, pp. 281-290, 2006.

[4] C. F. Gao, P. Tong, and T. Y. Zhang, "Interfacial crack problems in magneto-electroelastic solids," International Journal of Engineering Science, vol. 41, no. 18, pp. 2105-2121, 2003.

[5] C. F. Gao, P. Tong, and T. Y. Zhang, "Fracture mechanics for a mode II crack in a magneto-electro-elastic solid," International Journal of Solids and Structures, vol. 41, pp. 6613-6629, 2004.

[6] T. S. Cook and F. Erdogan, "Stresses in bonded materials with a crack perpendicular to the interface," International Journal of Engineering Science, vol. 10, no. 8, pp. 677-697, 1972.

[7] F. Erdogan and T. S. Cook, "Antiplane shear crack terminating at and going through a bimaterial interface," International Journal of Fracture, vol. 10, no. 2, pp. 227-240, 1974.

[8] Q. H. Qin and S. W. Yu, "An arbitrarily-oriented plane crack terminating at the interface between dissimilar piezoelectric materials," International Journal of Solids and Structures, vol. 34, no. 5, pp. 581-590, 1997.

[9] X. F. Li and B. L. Wang, "Anti-plane shear crack normal to and terminating at the interface of two bonded piezoelectric ceramics," International Journal of Solids and Structures, vol. 44, no. 11-12, pp. 3796-3810, 2007.

[10] H. F. Bueckner, "On a class of singular integral equations," Journal of Mathematical Analysis and Applications, vol. 14, no. 3, pp. 392-426, 1966.

[11] F. G. Tricomi, Integral Equations, Dover Publications, New York, NY, USA, 1985.

[12] Y. E. Pak, "Crack extension force in a piezoelectric material," ASME Journal of Applied Mechanics, vol. 57, no. 3, pp. 863$869,1990$.

[13] B. Rogowski, "The mode III cracks emanating from an elliptical hole in the piezo-electro-magneto-elastic materials," Archive of Applied Mechanics, vol. 81, pp. 1607-1620, 2011.

[14] X. Wang and S. Yu, "Transient response of a crack in piezoelectric strip subjected to the mechanical and electrical impacts: mode-I problem," Mechanics of Materials, vol. 33, no. 1, pp. 11-20, 2001.

[15] B. Gu, S. W. Yu, and X. Q. Feng, "Transient response of an insulating crack between dissimilar piezoelectric layers under mechanical and electrical impacts," Archive of Applied Mechanics, vol. 72, no. 8, pp. 615-629, 2002.

[16] J. H. Huang and W. S. Kuo, "The analysis of piezoelectric/piezomagnetic composite materials containing ellipsoidal inclusions," Journal of Applied Physics, vol. 81, no. 3, pp. 13781386, 1997.

[17] A. R. Annigeri, N. Ganesan, and S. Swarnamani, "Free vibration behaviour of multiphase and layered magneto-electroelastic beam," Journal of Sound and Vibration, vol. 299, no. 1-2, pp. 44-63, 2007.

[18] Z. F. Song and G. C. Sih, "Crack initiation behavior in magnetoelectroelastic composite under in-plane deformation," Theoretical and Applied Fracture Mechanics, vol. 39, no. 3, pp. 189-207, 2003. 

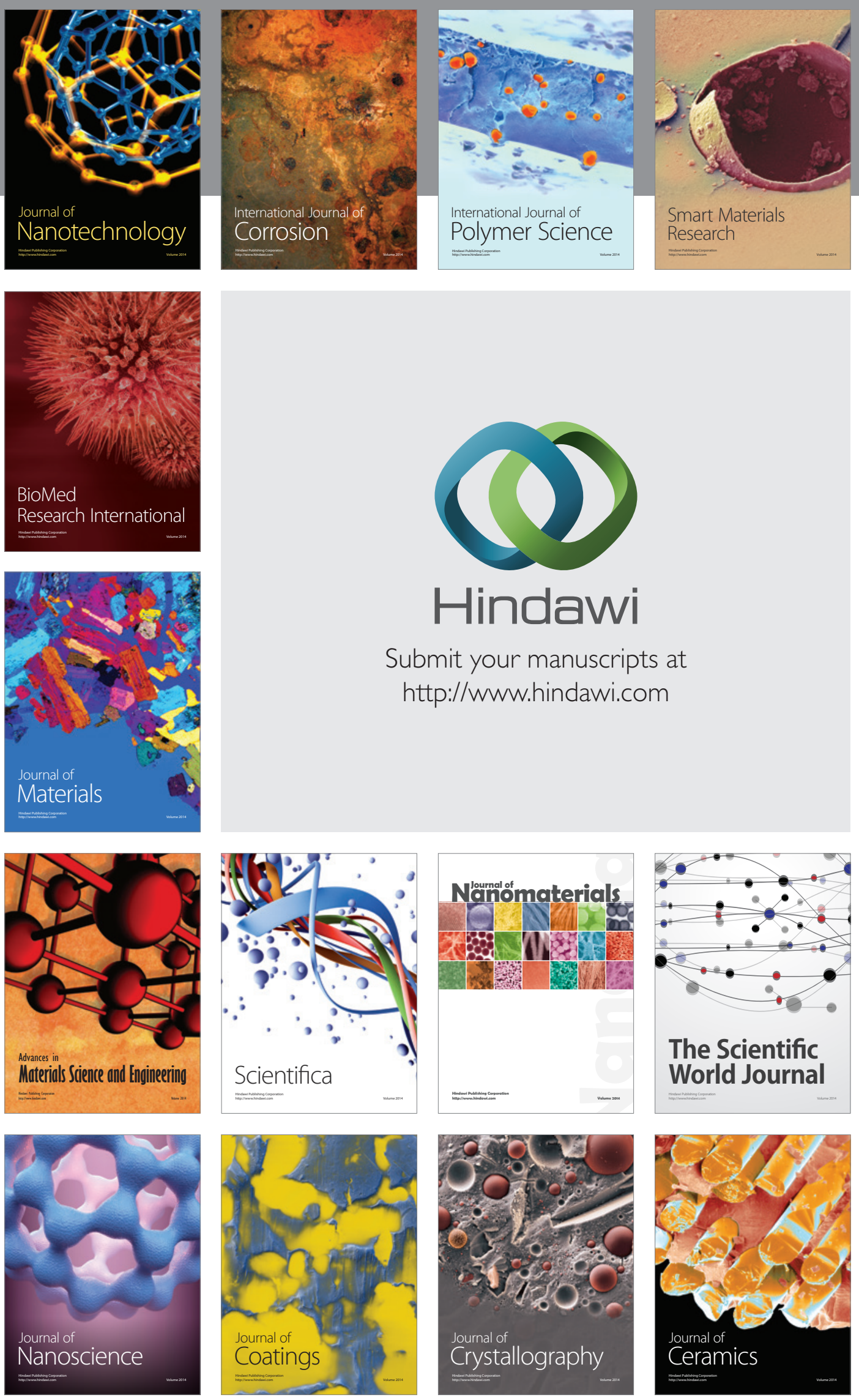

The Scientific World Journal

Submit your manuscripts at

http://www.hindawi.com

\section{World Journal}

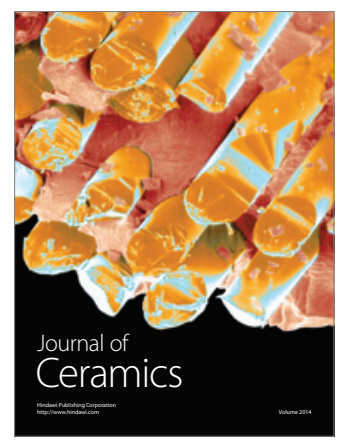

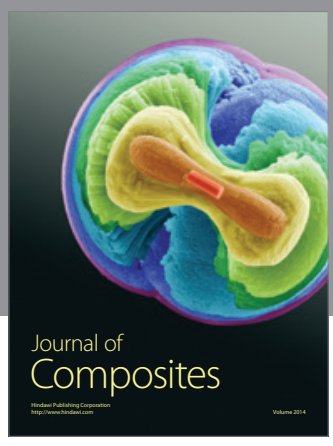
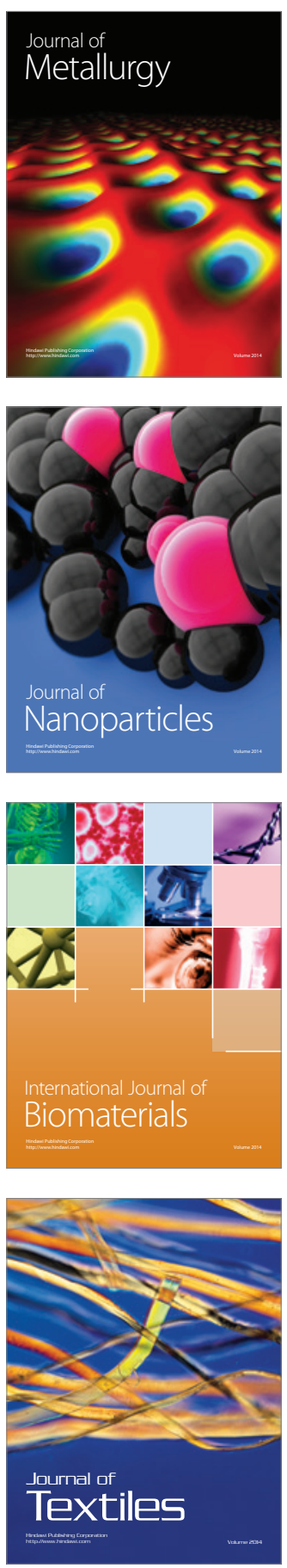